University of Pisa

\title{
On fields of algebraic numbers with bounded local degrees
}

\author{
$\mathrm{PhD}$ Thesis in Mathematics
}

Candidate: Sara Checcoli Advisor: Prof. Umberto Zannier 


\section{Introduction}

This work is about the relations between some properties which can occur for an infinite algebraic extension $K$ of the rationals.

The first property we shall consider is the uniformly boundedness of the local degrees of $K$, namely the existence of a constant $b$, depending only on the field $K$, such that for every prime number $p$ and every place $v_{p}$ of $K$ which extends the $p$-adic one, the completion of $K$ with respect to $v_{p}$ is a finite extension of $\mathbb{Q}_{p}$ of degree bounded by $b$.

The second property is the inclusion of the extension $K$ in a field $\mathbb{Q}^{(d)}$, which is defined as the compositum, in the algebraic closure of the rationals, of all number fields of degree at most $d$ over $\mathbb{Q}$.

The inquiry into the relation between these two properties was motivated by Bombieri and Zannier's paper [2], which studies the Northcott property (of the finiteness of elements of bounded absolute Weil height) for certain infinite extensions of $\mathbb{Q}$. The paper considers in particular the field $\mathbb{Q}^{(d)}$ and the Northcott property is proved for the compositum of all abelian extensions of $\mathbb{Q}$ of bounded degree, while for $\mathbb{Q}^{(d)}$ the question remains open. In this proof a crucial role is played by the uniform boundedness of the local degrees of $\mathbb{Q}^{(d)}$.

The considerations made in [2] motivated an investigation on the structure of the field $\mathbb{Q}^{(d)}$ and of fields with uniformly bounded local degrees. This led to the question, not free of independent interest, of whether every algebraic extension $K$ of $\mathbb{Q}$ with uniformly bounded local degrees is contained in $\mathbb{Q}^{(d)}$ for some positive integer $d$. More generally, are there properties which are equivalent to having uniformly bounded local degrees for an algebraic extension of the rationals?

The study of the structure of $\mathbb{Q}^{(d)}$ revealed some unexpected sides. These arise when we consider a third property for an infinite algebraic extension $K$ of $\mathbb{Q}$, that is whether every finite subextension of $K$ can be generated by elements of degree bounded by a constant depending only on $K$. A field with this property is certainly contained in $\mathbb{Q}^{(d)}$ for some positive integer $d$ and one could ask whether this condition is equivalent to being a subfield of $\mathbb{Q}^{(d)}$.

In this work we give a complete answer to all these questions proving the following result.

Theorem 1. Let $K / \mathbb{Q}$ be an infinite Galois extension. Then the following conditions are equivalent: 
(1) K has uniformly bounded local degrees at every prime;

(2) K has uniformly bounded local degrees at almost every prime;

(3) $\operatorname{Gal}(K / \mathbb{Q})$ has finite exponent.

Moreover, if $K / \mathbb{Q}$ is abelian, then the three properties:

(a) K has uniformly bounded local degrees;

(b) $K$ is contained in $\mathbb{Q}^{(d)}$ for some positive integer $d$;

(c) every finite subextension of $K$ can be generated by elements of bounded degree;

are equivalent. However, in general, (c) implies (b) which implies (a) and none of the inverse implications holds.

This work is structured in the following way.

In Chapter 1 we recall the basic concepts of the theory of $p$-adic fields. We start with the definition of valuations and completions and we discuss the main properties of $p$-adic fields, with special emphasis on the structure of their Galois extensions. The chapter ends with a brief overview on some basic results from local class field theory and a theorem of Shafarevich on the number of generators of the Galois group of a $p$-extension of a $p$-adic field. This result turns out to be very useful while looking for a bound on the local degrees of an algebraic extension of $\mathbb{Q}$.

Chapter 2 focuses on the study of the structure of infinite algebraic extensions of the rationals with uniformly bounded local degrees. In particular we concentrate on the field $\mathbb{Q}^{(d)}$, which has uniformly bounded local degrees, as is well known. This result belonged together with the motivations for the set questions and we recall its proof in Section 2.1. In Section 2.2 we give the first original result of this work: we investigate the structure of Galois extensions with uniformly bounded local degrees and we prove that these are exactly those whose Galois group has finite exponent. Moreover we prove that the existence of a uniform bound for the local degrees at almost every prime implies the uniform boundedness at every prime. The main ingredients of the proof are: an application of Chebotarev's density theorem; a result of Shafarevich on the number of generators of the Galois group of a $p$-extension of $p$ adic fields; Zelmanov's result on the Restricted Burnside Problem on the existence of a bound for the order of a finite and periodic group in terms of the number of its generators and of its exponent. In Section 2.3 we also provide a formula to compute an explicit bound for the local degrees of an extension $K$ of finite exponent in some special cases.

Finally in Section 2.4 we discuss the case of abelian extensions, proving the first part of Theorem 1 .

In order to give a complete proof of Theorem 1, we need to introduce some $p$-groups called extraspecial groups, namely finite $p$-groups in which the center and the commutator subgroup have both order $p$. These groups in fact shall 
enable us to construct examples of fields entailing the validity of the statements of Theorem 1. Extraspecial groups are classical objects in the theory of finite groups and they have been largely studied. In Chapter 3 we recall and prove the main properties of these groups and, in particular, we give a classification theorem for them. This classification is made in several steps. We first describe, in Section 3.1, the connection between symplectic spaces and extraspecial groups. Secondly, in Section 3.2, we introduce the concept of central product with amalgamated center and we prove that every extraspecial $p$-group is a central product with amalgamated centers of extraspecial groups of order $p^{3}$. As a consequence, we can prove the structure theorem for extraspecial groups. In particular we shall see that, if $p$ is an odd prime, every extraspecial $p$-group is uniquely determined, up to isomorphisms, by its order and its exponent. The chapter ends with the description of some irreducible modules of extraspecial groups and a property, which shall be used in our constructions, on the intersection of subgroups of bounded index of an extraspecial group.

In Chapter 4 we state and prove the main theorem of this work. The proof is divided into four parts: in the first part we give a condition for an infinite Galois extension $K$ of the rationals to be contained in $\mathbb{Q}^{(d)}$ based on the existence of some minimal normal subgroups of the Galois groups of finite Galois subextensions of $K$. In the remaining parts, we prove the non equivalence of properties described in Theorem 1: the proof is based on some group-theoretical constructions with extraspecial groups and their modules, followed by an application of Shafarevich's Theorem about the realization of solvable groups as Galois groups. We also compute explicit bounds for the local degrees of certain extensions, improving the bounds given by the formula of Section 2.3.

The use of Shafarevich's Theorem can actually be avoided by an explicit realization of the groups constructed in Chapter 4 and this will be done in Chapter 5, where we concretely construct Galois extension with the sought for Galois groups. We first describe how to realize extraspecial groups of order $p^{3}$ and then, using the structure theorem for these groups, we give realizations of extraspecial groups of any order. Finally we shall realize the semidirect product of an extraspecial group by its group algebra over certain finite fields. These constructions are based on some elementary methods in inverse Galois theory and on a result by Serre.

\section{Acknowledgments}

Completing a thesis is a challenge, thanking all those who contributed to it is an even greater one. Many people were involved in one or the other way in it. I want to thank all of them, including those not mentioned here by name.

I would like to express my gratitude to my $\mathrm{PhD}$ supervisor, Prof. Umberto Zannier, whose help and expertise added considerably to my graduate experience.

I am also grateful to many other people for useful mathematical discussions during the course of this work; I wish to thank in particular Prof. Roberto 
Dvornicich and Maurizio Monge. A very special thank goes to Prof. Andrea Lucchini for introducing me to the world of extraspecial groups and for his kind help and support.

I want to thank the director of the PhD school Prof. Fabrizio Broglia, for helping me a lot and for being a reference point throughout my $\mathrm{PhD}$.

I would like to thank the many people who have taught me mathematics: my undergraduate teachers at Bologna (especially Luca Migliorini and Angelo Vistoli), and my graduate teachers (especially Alessandro Languasco, Marco Garuti, Gilles Zémor and Adrian Iovita). For their kind assistance with writing letters, giving wise advice, helping with various applications, and so on, I wish to thank in addition Giovanni Alberti and, again, Andrea Lucchini.

I am indebted to many people at the Department of Mathematics in Pisa for providing a stimulating and fun environment in which to learn and grow. I am especially grateful to Giorgio, Enrico, Pietro, Bruno, Riccardo, Fulvio and my officemates Isaia, Fionntann, John and Tiziano for their support, their kindness, their help, all the time spent together and for making Pisa a beautiful place to study.

I wish to thank all my friends from Bologna ( Giulia, Chiara, Rya, Dilli and all the pinguine), from Padova (Alessandro, Marco, Vale), from Paris (Pietro, Federica, Stefania and all 8 Rue d'Avron) for all the emotional support, entertainment, and caring they provided.

I wish to thank Laura for making my last months in Pisa something which I will miss a lot and I thank Luca for everything.

Lastly, and most importantly, I want to thank my extended family: Mari and Fritz, my family in Pisa, for their support, help and for these three unique years; Maurizio, Patrizia, Marta and Andrea for being always there and for their uniformly unbounded love. This work would not have been possible without them; to them I dedicate this thesis. 


\section{Contents}

1 Preliminaries on $p$-adic fields 9

1.1 Absolute values and valuations . . . . . . . . . . . . . . . . 9

1.2 Completions .......................... 11

$1.3 p$-adic fields . . . . . . . . . . . . . . . . . . . . 14

1.4 Extensions of $p$-adic fields . . . . . . . . . . . . . . . 16

(1) Unramified extensions . . . . . . . . . . . . . . . 16

(2) Totally ramified extensions . . . . . . . . . . . . . . . . . 17

1.5 The Galois group of a $p$-adic fields extension . . . . . . . . . . . . 19

1.6 Shafarevich's theorem on $p$-extensions . . . . . . . . . . . . 20

2 Galois extensions with finite exponent 23

2.1 Definitions and motivations . . . . . . . . . . . . . . 24

2.2 Characterization of fields with bounded local degrees . . . . . . . 26

2.3 A formula for bounding the local degrees . . . . . . . . . . . . . 28

2.4 The abelian case . . . . . . . . . . . . . . . 30

3 Extraspecial groups $\quad 33$

3.1 Definitions and first properties . . . . . . . . . . . . . 34

3.1.1 Symplectic spaces and quadratic forms . . . . . . . . . 35

3.1.2 Connection between symplectic spaces and extraspecial

p-groups ...................... 36

3.2 Classification of extraspecial p-groups . . . . . . . . . . . . 39

3.2.1 Central products of groups amalgamated on the centers . 39

3.2.2 Extra-special $p$-groups of order $p^{3}$. . . . . . . . . . . 41

3.2.3 The structure theorem for extraspecial $p$-groups . . . . . 44

3.3 Some irreducible modules . . . . . . . . . . . . . . . . 46

3.4 A special property . . . . . . . . . . . . . . . . 47

4 The main theorem $\quad \mathbf{4 9}$

4.1 Statement of the theorem . . . . . . . . . . . . . . 49

4.2 Proof of $(2) \ldots \ldots \ldots \ldots$

4.3 Proof of $(\mathrm{i}) \ldots \ldots \ldots \ldots \ldots \ldots$

4.4 Proof of $($ ii $) \ldots \ldots \ldots \ldots \ldots$. . . . . . . . . . . . . . . . . . . . . . . . . . . . . . . . 51

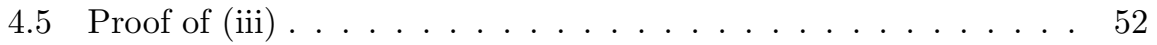


4.5.1 Construction of the field $K$. . . . . . . . . . . . . . 53

4.5.2 An explicit bound for the local degrees of $K \ldots \ldots$. . . . 54

5 Some explicit constructions $\quad \mathbf{5 7}$

5.1 Realizing extraspecial groups ... . . . . . . . . . . . . 57

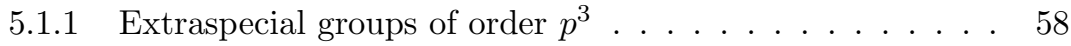

5.1 .2 Extraspecial groups of any order and exponent $p \ldots 62$

5.2 Extension by the group algebra . . . . . . . . . . . . . . 64

Bibliography ........................... 68 


\section{Chapter 1}

\section{Preliminaries on $p$-adic fields}

This chapter provides some important tools from the theory of $p$-adic fields, which are among the main objects of study of this work; we shall recall some notions and properties concerning $p$-adic fields, developing the theory only to such an extent as is indeed for the purposes of this work.

We will omit most of the proofs of the results stated, for which the interested reader should see Narckiewicz's book [9] or any other classical introductory book on Algebraic Number Theory.

The chapter has the following structure: in Section 1.1 we recall the definitions of absolute value and valuation and we describe in Section 1.2 the completion of a field with respect to a valuation. In Section 1.3 we focus on $p$-adic fields, namely completions of number fields with respect to valuations above $p$ and we describe their structure. Section 1.4 is devoted to the study of extensions of $p$-adic fields: we recall the main properties of unramified extensions and totally ramified extensions. In Section 1.5 we concentrate on the structure of the Galois group of a Galois extension of $p$-adic fields. Finally, in Section 1.6 , we mention some basic results from local class field theory and we prove a theorem of Shafarevich on the number of generators of the Galois group of a $p$-extension of $p$-adic fields. This result will become central in the search for a bound for the local degrees of an infinite algebraic extension of the rationals.

We assume the reader to be familiar with the theory of number fields, splitting of primes in extensions and basic Galois Theory for finite and infinite extensions of fields.

\subsection{Absolute values and valuations}

We start this section by listing the fundamental definitions and properties which will be needed in this chapter. 
Definition 1.1. Let $K$ be a field. We call an absolute value of $K$ a function $|\cdot|: K \rightarrow \mathbb{R}_{\geq 0}$ satisfying the following properties:

(i) $|x|=0 \Leftrightarrow x=0$;

(ii) $|x y|=|x| \cdot|y|$ for all $x, y \in K$;

(iii) $|x+y| \leq|x|+|y|$ for all $x, y \in K$.

An absolute value is called non-archimedean if it also satisfies the stronger inequality $|x+y| \leq \max \{|x|,|y|\}$ for all $x, y \in K$ and it is called archimedean otherwise.

Two absolute values $|\cdot|_{1}$ and $|\cdot|_{2}$ of $K$ are said to be equivalent if there exists a constant $c \in \mathbb{R}_{>0}$ such that $|x|_{1}=|x|_{2}{ }^{c}$ for all $x \in K$. An equivalence class of absolute values of $K$ is called a place of $K$.

Given an absolute value $|\cdot|$ on a field $K$, one can define a metric on $K$ induced by $|\cdot|$, setting $d(x, y)=|x-y|$ for all $x, y \in K$, which makes $K$ a topological field (that is, addition and multiplication in $K$ are continuous functions under the topology associated to the induced metric). It is easy to prove that two absolute values of $K$ are equivalent if and only if they induce the same topology on $K$ (see [9], Ch. 1, §3, Prop. 1.13). We now define a concept which is strictly connected to that of absolute value.

Definition 1.2. Let $K$ be a field. A (real) valuation of $K$ is a homomorphism $v: K \rightarrow \mathbb{R} \cup\{\infty\}$ with the properties

(i) $v(x)=\infty \Leftrightarrow x=0$;

(ii) $v(x y)=v(x)+v(y)$;

(iii) $v(x+y) \geq \min \{v(x), v(y)\}$.

A field $K$ with a valuation $v$ is called a valued field and is denoted by $(K, v)$.

A valuation is said to be discrete if $v\left(K^{*}\right)$ is a discrete subgroup of $\mathbb{R}$. Two valuations $v_{1}$ and $v_{2}$ of $K$ are equivalent if there exists a positive real number $r$ such that $v_{1}(x)=r v_{2}(x)$ for every $x \in K$.

Remark 1.1. There is a one-to-one correspondence between the set of equivalence classes of valuations of a field $K$ and the set of equivalence classes of non-archimedean absolute values of $K$. This correspondence is given by the following associations. If $v$ is a valuation and we fix a real number $t$ with $0<t<1$, the map defined as $|x|_{v}:=t^{v(x)}$ if $x \neq 0$ and $|0|_{v}:=0$ is a non-archimedean absolute value on $K$. On the other hand, if $|\cdot|$ is a non-archimedean absolute value on $K$ and we fix $b \in \mathbb{R}_{>1}$, the map $v$, defined setting $v(x):=-\log _{b}|x|$ for $x \neq 0$ and $v(0):=\infty$, is a valuation on $K$. It is easy to check that these associations induce the sought for correspondence between equivalence classes of absolute values and valuations and that this does not depend on the choice of the constants $t$ and $b$. 
We now make some classical examples that we shall largely discuss in the sequel.

Example 1.1. Let $K=\mathbb{Q}$ and $p$ be a prime number. Every $x \in \mathbb{Q}$ can be written in a unique way as $x=p^{r}(m / n)$ where $r, m$ and $n$ are integers, $p, m$ and $n$ are coprime and $n$ is non negative.

We define a non-archimedean absolute value $|\cdot|_{p}$ and a valuation $v_{p}$ on $\mathbb{Q}$ setting $|x|_{p}:=p^{-r}$ and $v_{p}(x):=r$. The maps $|\cdot|_{p}$ and $v_{p}$ are called the $p$-adic absolute value and the $p$-adic valuation respectively.

On $\mathbb{Q}$ we also have the usual archimedean absolute value $|\cdot|_{\infty}$ defined as $|x|_{\infty}:=x$ if $x \geq 0$ and $|x|_{\infty}:=-x$ if $x<0$.

An important theorem by Ostrowski states that, up to equivalences, these are the only absolute values on $\mathbb{Q}$ (Ostrowski's result is more general and it classifies valuations over number fields, see Example 1.2 below).

Example 1.2. Let $K$ be a number field and denote by $\mathcal{O}_{K}$ its ring of integers. Let $\wp \subset \mathcal{O}_{K}$ be a prime ideal of $K$. We define a valuation $v_{\wp}$ of $K$ associated to $\wp$ as it follows: if $x \in K$, we consider the ideal generated by $x$ and we take its prime factorization in $\mathcal{O}_{K}$, that is

$$
(x)=\prod_{i=1}^{n} \wp_{i}^{a_{i}}
$$

where $a_{i} \in \mathbb{Z}$. Then we set $v_{\wp}(x):=a_{i}$ if $\wp=\wp_{i}$ for some index $i$ and $v_{\wp}(x):=0$ otherwise.

Now the ideal $\wp$ lies above some prime $p$ of $\mathbb{Q}$ (that is above the ideal generated by $p$ ) and, up to multiplication by an integer, $\left.v_{\wp}\right|_{\mathbb{Q}}=v_{p}$; in this case we say that the valuation $v_{\wp}$ extends the $p$-adic valuation to $K$ or that $v_{\wp}$ is a valutation above $p$.

By Ostrowski's theorem (see [9], Ch. 3, §1, Thm. 3.2) these are the only non-archimedean absolute values of $K$ and different primes of $\mathcal{O}_{K}$ define non equivalent valuations. Moreover every archimedean absolute value of $K$ comes from an embedding of $K$ into the complex field. If $\sigma$ is such an embedding, the valuation associated to it is defined as $v_{\sigma}(x):=|\sigma(x)|$ for all $x \in K$, where $|\cdot|$ is the usual complex absolute value. Two archimedean absolute values defined by different embeddings are equivalent if and only if those embeddings are complex conjugated.

\subsection{Completions}

We start this section by recalling the definition of valuation ring.

Definition 1.3. Let $K$ be a field. A valuation ring of $K$ is a subring $A$ of $K$ such that for every $x \in K$ either $x \in A$ or $x^{-1} \in A$.

It is easy to see that if $A$ is a valuation ring of a field $K$, then $K=\operatorname{Frac}(A)$ is the field of fractions of $A$ and the ideal $m:=\left\{x \in A \mid x^{-1} \notin A\right\}$ is the only maximal ideal of $A$. We have the following proposition. 
Proposition 1.2.1. Let $K$ be a field and $v$ a valuation of $K$. Then

$$
R_{v}:=\left\{x \in K^{*} \mid v(x) \geq 0\right\} \cup\{0\}
$$

is a valuation ring of $K$ with maximal ideal

$$
m_{v}:=\left\{x \in K^{*} \mid v(x)>0\right\} \cup\{0\} .
$$

Moreover $v$ is discrete if and only if $m_{v}$ is principal. In this case $m_{v}$ is generated by an element of minimal positive valuation called uniformizer and $R_{v}$ is a principal ideal domain.

Proof. See [9], Ch. 1, §3, Thm. 1.13.

Definition 1.4. $R_{v}$ is called the valuation ring of $K$ with respect to $v$ and $\kappa_{v}:=R_{v} / m_{v}$ is a field called the residue field of $K$ at $v$.

Now, given a field together with an absolute value $|\cdot|$, we want to define the completion of this field with respect to the topology induced by $|\cdot|$. We have the following fundamental theorem.

Theorem 1.2.2. Let $K$ be a field and $|\cdot|$ be an absolute value of $K$. Then there exists a field $K^{\prime}$ and an absolute value $\|\cdot\|$ of $K^{\prime}$ such that:

(1) $K^{\prime}$ is complete for the topology induced by $\|\cdot\|$;

(2) $K \subseteq K^{\prime}$ and the restriction of $\|\cdot\|$ to $K$ equals $|\cdot|$;

(3) $K$ is dense in $K^{\prime}$.

A field $K^{\prime}$ with these properties is unique up to (algebraic and topological) isomorphisms.

Proof. The result follows easily by viewing $K^{\prime}$ as the quotient of the ring of Cauchy's sequences of $K$ with respect to $|\cdot|$ by its maximal ideal of all nullsequences with respect to $|\cdot|$. One proves that this construction gives the sought for object (see for instance [9], Ch. 3, §1, Thm. 3.1).

Definition 1.5. The field $K^{\prime}$ of Theorem 1.2.2 is called the completion of $K$ with respect to $|\cdot|$.

If $|\cdot|$ is non-archimedean and corresponds to the valuation $v$, we call $K^{\prime}$ the completion of $K$ with respect to $v$ and we denote it by $K_{v}$.

The following propositions relates the structure of the valuation ring of a valued field with the valuation ring of its completion.

Proposition 1.2.3. Let $(K, v)$ be a valued field with valuation ring $R_{v}$ and maximal ideal $m_{v}$. Let $K^{\prime}$ be the completion of $K$ with respect to $v$ and $R^{\prime}$ and $m^{\prime}$ be its ring of valuation and the maximal ideal. Then $R^{\prime}$ is the closure of $R_{v}$ and $m^{\prime}$ is the closure of $m_{v}$. Moreover if $v$ is discrete we have that: 
1. the residue fields of $K$ and $K^{\prime}$ are isomorphic and if $m_{v}$ is principal, so is $\mathrm{m}^{\prime}$;

2. if $m_{v}$ is generated by an element $\pi$ of minimal valuation, we have

$$
\begin{aligned}
R^{\prime} & =\left\{\sum_{i=0}^{\infty} a_{i} \pi^{i} \mid a_{i} \in R \text { represents an element of } R_{v} / m_{v}\right\} \\
R^{*} & =\left\{\sum_{i=0}^{\infty} a_{i} \pi^{i} \mid a_{0} \neq 0, a_{i} \text { as before }\right\} \\
m^{\prime} & =\left\{\sum_{i=1}^{\infty} a_{i} \pi^{i} \mid a_{i} \text { as before }\right\} \\
K^{\prime} & =\left\{\sum_{i=n}^{\infty} a_{i} \pi^{i} \mid n \in \mathbb{Z}, a_{i} \text { as before }\right\}
\end{aligned}
$$

3. if $\left|R_{v} / m_{v}\right|$ is finite, then $R^{\prime}=\lim _{n} R_{v} / m_{v}{ }^{n}$ (the inverse limit being taken under the projection maps).

Proof. See [9], Ch. 3, §1, Corollaries 1, 2 and 3.

Example 1.3. Let $K=\mathbb{Q}$. The completion of $\mathbb{Q}$ with respect to the archimedean absolute value is the field of real numbers $\mathbb{R}$.

We now describe the completion with respect to a non-archimedean absolute value, that is, from previous considerations, a $p$-adic valuation $v_{p}$, where $p$ is a prime number. We have:

- $R_{v_{p}}=\mathbb{Z}_{(p)}=\left\{\frac{a}{b} \in \mathbb{Q} \mid b \notin(p)\right\}$ is the localization of $\mathbb{Z}$ at $(p)$;

- $m_{v_{p}}=p \mathbb{Z}_{(p)}$ is generated by the element $\pi=p$ of minimal valuation $v_{p}(p)=1$;

- $\kappa_{v_{p}}=R_{v_{p}} / m_{v_{p}}=\mathbb{Z}_{(p)} / p \mathbb{Z}_{(p)} \simeq \mathbb{Z} / p \mathbb{Z}$ is a finite field.

The completion of $\mathbb{Q}$ with respect to the $p$-adic valuation is called the field of p-adic numbers $\mathbb{Q}_{p}$ and the closure of $\mathbb{Z}_{(p)}$ in $\mathbb{Q}_{p}$ is the ring of $p$-adic integers denoted by $\mathbb{Z}_{p}$. The maximal ideal of $\mathbb{Z}_{p}$ is $p \mathbb{Z}_{p}$ and, in view of Proposition 1.2.3, we have

$$
\mathbb{Z}_{p}=\left\{\sum_{i=0}^{\infty} a_{i} p^{i} \mid a_{i} \in\{1, \ldots, p\}\right\} \simeq \underset{\overleftarrow{n}}{\lim } \mathbb{Z} / p^{n} \mathbb{Z}
$$

We end this section by listing some important properties on the behaviour of valuations in normal extensions. We have the following result.

Proposition 1.2.4. Let $K$ be a field of characteristic zero which is complete with respect to an absolute value $|\cdot|$ and has finite residue field. Let $F / K$ be a finite extension of degree $n$. Then $|\cdot|$ extends in a unique way to $F$ by an 
absolute value $\|\cdot\|$ defined as $\|x\|:=\left|N_{F / K}(x)\right|^{1 / n}$ for all $x \in F$, where $N_{F / K}$ is the norm function of the extension $F / K$. Moreover $F$ is complete with respect to the absolute value $\|\cdot\|$.

Proof. See [9], Ch. 5, §1, Prop. 5.3.

As a corollary we have the following:

Corollary 1.2.5. Let $K$ be a field complete with respect to an absolute value $|\cdot|$ and denote by $K^{\text {alg }}$ an algebraic closure of $K$. Then the absolute value $|\cdot|$ extends in a unique way to an absolute value $\|\cdot\|$ of $K^{\text {alg }}$.

Proof. For every $x \in K^{\text {alg }}$ we define $\|x\|:=\left|N_{K(x) / K}(x)\right|$.

\section{$1.3 \quad p$-adic fields}

This section focuses on the structure and properties of finite algebraic extensions of $\mathbb{Q}_{p}$. We start with the following definition.

Definition 1.6. A $p$-adic field is the completion of a number field with respect to a discrete valuation $v$ extending the $p$-adic valuation of $\mathbb{Q}$.

We will sometimes refer to these fields as to local fields (usually this terminology denotes, more in general, the completion of a global field, that is either a number field or a function field in one variable over a finite field, with respect to some absolute value).

We want to study in detail the structure of $p$-adic fields. In order to do this we fix some notation.

Let $L$ be a number field of degree $n$ over $\mathbb{Q}$ and denote by $\mathcal{O}_{L}$ its ring of integers. As explained in Example 1.2, every discrete valuation of $L$ is induced by a prime ideal $\wp$ of its ring of integers and we denote it by $v_{\wp}$. We denote by $L_{\wp}$ the completion of $L$ with respect to $v_{\wp}$ and by $R_{\wp}$ and $m_{\wp}$ the valuation ring of $L_{\wp}$ and its maximal ideal respectively. We recall that, in view of Proposition 1.2.3, $R_{\wp}$ is the closure of $\mathcal{O}_{L}$ in $L_{\wp}$ and the residue field $\kappa_{L}=R_{\wp} / m_{\wp}$ is isomorphic to $\mathcal{O}_{L} / \wp$.

The following theorem enables us to translate many problems concerning finite extensions of algebraic number fields into the language of $p$-adic fields.

Theorem 1.3.1. Let $L / K$ be an extension of number fields. Let $\wp \subset \mathcal{O}_{K}$ be a prime of $K$ such that $\wp \mathcal{O}_{L}=\beta_{1}^{e_{1}} \ldots \beta_{r}^{e_{r}}$ where the $\beta_{i}$ 's are distinct primes of $L$ and $e_{i}=e_{L / K}\left(\beta_{i}\right)$ and $f_{i}=f_{L / K}\left(\beta_{i}\right)$ are the ramification index and the inertia degree of $\beta_{i}$ in the extension $L / K$ respectively. Then we have:

1. $L_{\beta_{i}}=K_{\wp} L$ is generated by $L$ and $K_{\wp}$ (as subfields of $\left.L_{\beta_{i}}\right)$;

2. $\left[L_{\beta_{i}}: K_{\wp}\right]=e_{i} f_{i}$;

3. $R_{\beta_{i}}$ is the integral closure of $R_{\wp}$ in $L_{\beta_{i}}$; 
4. $m_{\beta_{i}}$ is the only prime ideal of $R_{\beta_{i}}$ lying above $m_{\wp}$ and the inertia degree and ramification index of $m_{\beta_{i}}$ in the extension $L_{\beta_{i}} / K_{\wp}$ are equal to those of $\beta_{i}$ in the extension $L / K$;

5. the residue field $\kappa_{\beta_{i}}=R_{\beta_{i}} / m_{\beta_{i}}$ is a finite extension of the residue field $\kappa_{\wp}=R_{\wp} / m_{\wp}$ of degree $f_{i}$.

Proof. See [9], Ch. 5, §1, Thm. 5.5.

Remark 1.2. From Theorem 1.3 .1 with $K=\mathbb{Q}$, we have that the completion of a number field $L$ with respect to a discrete valuation induced by some prime ideal is always a finite extension of $\mathbb{Q}_{p}$ for some prime number $p$, which motivates the name $p$-adic for these fields. Moreover the residue field of $L$ with respect to this valuation is a finite field of characteristic $p$, that is a finite extension of the finite field $\mathbb{F}_{p}$.

The following definition will be very important in the ensuing chapters.

Definition 1.7. Let $L$ be a number field and $v$ a valuation of $L$ extending the $p$-adic one. We call the local degree of $L$ at $v$ the degree of the extension $L_{v} / \mathbb{Q}_{p}$.

Remark 1.3. It follows easily from the definition and Theorem 1.3 .1 that for a number field $L$ the local degree at every valuation is bounded by $[L: \mathbb{Q}]$.

We end this section with two results which are very important in the theory of $p$-adic fields. The first result is known as Krasner's lemma.

Lemma 1.3.2 (Krasner's lemma). Let $K$ be a field which is complete with respect to a non-archimedean absolute value $|\cdot|$. Let $\alpha, \beta \in K^{\text {alg }}$ be two elements in a fixed agebraic closure of $K$. Suppose moreover that $\alpha$ is separable over $K(\beta)$. If $|\alpha-\beta|<|\sigma(\alpha)-\alpha|$ for every embedding $\sigma \neq 1$ of $K(\alpha)$ into $K^{\text {alg }}$, then $K(\alpha) \subseteq K(\beta)$.

Proof. One has to show that $[K(\alpha, \beta): K(\beta)]=1$. Since by assumption $\alpha$ is separable over $K(\beta)$ this is equivalent to prove that if $\rho: K(\alpha, \beta) \hookrightarrow K^{\text {alg }}$ is an embedding which is the identity on $K(\beta)$, then $\rho(\alpha)=\alpha$. Now

$$
|\rho(\alpha)-\alpha|=|\rho(\alpha)-\rho(\beta)+\beta-\alpha|<|\sigma(\alpha)-\alpha|
$$

and since this holds for every $\sigma$, we must have $\rho(\alpha)=\alpha$.

Using Krasner's lemma it can be proved that two monic irreducible polynomials of the same degree whose corresponding coefficients are sufficiently close to each other define the same extension, as stated in the following corollary. This result can be used to prove that a $p$-adic field has only a finite number of extensions of a given degree, as we shall see in Section 2.1.

Corollary 1.3.3. Let $K$ be complete with respect to a discrete valuation $v$, let $R$ be its valuation ring and $m$ its maximal ideal. Assume moreover that the residue field $R / m$ is finite. Consider two monic polynomials in $R[t]$

$$
F(t)=t^{n}+a_{n-1} t^{n-1}+\ldots+a_{0}
$$


and

$$
G(t)=t^{n}+b_{n-1} t^{n-1}+\ldots+b_{0}
$$

with $F(t)$ irreducible over $K$. Suppose that, for every $i, a_{i}$ and $b_{i}$ are sufficiently closed. Then $G(t)$ is also irreducible over $K$ and for every root $\alpha \in K^{\text {alg }}$ of $F(t)$, there exists a root $\beta \in K^{\text {alg }}$ of $G(t)$ such that $K(\alpha)=K(\beta)$.

Proof. See [9], Ch. 5, §1, Prop. 5.5.

\subsection{Extensions of $p$-adic fields}

We now describe some properties of the structure of an extension of $p$-adic fields. Before we state them, we recall the definition of compositum of fields. This definition is very important in this work, since we shall mainly deal with fields obtained as a compositum of other fields.

Definition 1.8. Let $\mathcal{F}$ be a family of fields and suppose that all elements of $\mathcal{F}$ are contained in a bigger field $F$. The compositum of the family $\mathcal{F}$ is the smallest subfield of $F$ containing all the elements of the family.

We now let $L / K$ be a finite extension of $p$-adic fields of degree $n$ and we denote by $\kappa_{L}$ and by $\kappa_{K}$ and by $R_{L}$ and $R_{K}$ the residue fields and rings of integers of $L$ and $K$ respectively. Then $\kappa_{L}$ and $\kappa_{K}$ are finite fields of characteristic $p$ and, from Theorem 1.3.1, we know that $n=e_{L / K} f_{L / K}$ where $e_{L / K}$ is the ramification index of $L / K$ and $f_{L / K}=\left[\kappa_{L}: \kappa_{K}\right]$. We have the following definition.

Definition 1.9. An extension of $p$-adic fields $L / K$ is said to be:

(1) unramified if $e_{L / K}=1$;

(2) totally ramified if $f_{L / K}=1$;

(3) tamely ramified if $p$ does not divide $e_{L / K}$;

(4) wildly ramified if $p$ divides $e_{L / K}$.

We start with the description of unramified extensions; then we shall discuss the totally ramified case.

\section{(1) Unramified extensions}

We now list some properties of unramified extensions which clarify how they can be generated.

Proposition 1.4.1. For a finite extension of $p$-adic fields $L / K$ the following conditions are equivalent:

1. $L / K$ is unramified; 
2. $L=K(\alpha)$ for some $\alpha \in R_{L}$ such that the image of $\alpha$ in $\kappa_{L}$ is a simple root of a polynomial $\bar{\mu}(t) \in \kappa_{K}[t]$, obtained by reducing modulo $\wp$ the coefficient of some monic polynomial $\mu(t) \in R_{K}[t]$ with $\mu(\alpha)=0$, $\wp$ being the maximal ideal of $R_{K}$;

3. $L=K\left(\zeta_{m}\right)$ where $\zeta_{m}$ is a primitive $m$-th root of unity with $m$ not divisible by $p$.

Proof. See [9], Ch. 5, §2, Lemma 5.4 and Thm. 5.9.

Remark 1.4. In view of the previous proposition and using the multiplicative property of the ramification indexes, it is easy to prove that the compositum of two unramified extensions of $K$ is again an unramified extension of $K$.

Thus if $L / K$ is a finite extension of $p$-adic fields and we denote by $K^{\text {ur }}$ the compositum of all unramified extensions of $K$ contained in $L$, we have that $K^{\mathrm{ur}} / K$ is unramified and $L / K^{\mathrm{ur}}$ is totally ramified. These considerations lead to the following result:

Corollary 1.4.2. Every finite extension of p-adic fields can be decomposed into an unramified part and a totally ramified part.

The next result is about the structure of the Galois group of unramified extensions.

Proposition 1.4.3. Let $K$ be a p-adic field with residue field $\kappa_{K}$. Then for every finite extension $\kappa / \kappa_{K}$ there exists a unique unramified extension $L / K$ such that the residue field of $L$ is isomorphic to $\kappa$. Moreover the extension $L / K$ is normal and its Galois group is isomorphic to the cyclic group $\mathrm{Gal}\left(\kappa / \kappa_{K}\right)$.

Proof. See [9], Ch. 5, §2, Thm. 5.8.

Since a finite field has a unique extension of a given degree, as a corollary we have:

Corollary 1.4.4. For every $p$-adic field $K$ and every positive integer $n$ there exists exactly one unnramified extension of $K$ of degree $n$.

\section{(2) Totally ramified extensions}

We now focus on totally ramified extensions, i.e. extensions $L / K$ such that the $f_{L / K}=1$. We shall recall some results on the structure of these extensions and in particular on the polynomials generating them. We start with the following definition.

Definition 1.10. Let $R$ be a Dedekind domain. An Eisenstein's polynomial over $R$ is a polynomial

$$
f(t)=a_{n} t^{n}+\ldots+a_{1} t+a_{0} \in R[t]
$$

for which there exists a prime $\wp \subset R$ such that $a_{n} \notin \wp, a_{i} \in \wp$ for $i \neq n$ and $a_{0} \in \wp \backslash \wp^{2}$. 
We now have the following result.

Proposition 1.4.5. If $L / K$ is totally ramified, then there exists $\alpha \in R_{L}$ such that $R_{L}=R_{K}[\alpha]$ and the minimal polynomial of $\alpha$ over $K$ is an Eisenstein's polynomial. Moreover if $L$ is generated over $K$ by a root of an Eisenstein's polynomial, then $L / K$ is totally ramified.

Proof. See [9], Ch. 5, §2, Thm. 5.10.

We have the corollary:

Corollary 1.4.6. A p-adic field $K$ has totally ramified extensions of any given degree.

Proof. If $\pi$ generates the maximal ideal of $R_{K}$, then the family $\left\{t^{e}-\pi \mid e \in \mathbb{N}\right\}$ is a family of Eisenstein's polynomials in $R_{K}[t]$ and their roots generates totally ramified extensions of every given degree.

We shall now discuss the case of tamely ramified extensions which is easier to study than the wild case, thanks to the following proposition.

Proposition 1.4.7. Let $L / K$ be a totally ramified and tame extension and let $\pi$ be a generator for the maximal ideal $\wp$ of $R_{K}$. Then there exists an element $\pi_{L}$, which generates the maximal ideal of $R_{L}$, such that $\pi_{L}{ }^{e_{L} / K}=\pi$.

Moreover if $a \in R_{K}$ and $e$ is a positive integer prime to $p$, then every root of the polynomial $t^{e}-a \in R_{K}[t]$ generates a tame extension of $K$. This extension is totally ramified if $\left(e, v_{\wp}(a)\right)=1$ (where $v_{\wp}$ is the valuation of $K$ associated to the prime $\wp)$.

Proof. See [9], Ch. 5, §2, Thm. 5.11 and Lemma 5.5.

As for unramified extensions, we have that the compositum of tamely ramified extensions is again tamely ramified.

Proposition 1.4.8. Let $L / K$ be a finite extension of $p$-adic fields and let $K^{\text {tame }}$ be the compositum of all tamely ramified extensions of $K$ contained in $L$. Then $K^{\text {tame }} / K$ is tamely ramified and $L / K^{\text {tame }}$ is a wildly and totally ramified extension whose degree is a power of $p$.

Proof. See [9], Ch. 5, §2.2, Corollary 3.

Now we can describe the general structure of an extension of $p$-adic fields as it follows.

Proposition 1.4.9. Let $L / K$ be a finite extension of $p$-adic fields of degree $n=e_{L / K} f_{L / K}$ and suppose that $e_{L / K}=e_{0} p^{s}$ where $\left(e_{0}, p\right)=1$. Then there exist extensions $K \subseteq F \subseteq E \subseteq L$ such that:

- $F / K$ is unramified and cyclic of degree $f_{L / K}$;

- $E / F$ is totally ramified and tame of degree $e_{0}$;

- L/E is totally ramified of degree $p^{s}$.

Proof. We set $F=K^{\text {ur }}$ and $E=F^{\text {tame }}$ and we apply Remark 1.4 and Proposition 1.4.8. 


\subsection{The Galois group of a $p$-adic fields extension}

We shall now concentrate on the case of Galois extensions of $p$-adic fields. In this section the following notation will be used: if $L / K$ is a Galois extension, we denote by $\operatorname{Gal}(L / K)$ its Galois group. Moreover if $\beta$ is a prime of $L$ lying above a prime $\wp$ of $K$, we indicate with $D(\beta \mid \wp)$ the decomposition group of $\beta$, namely the subgroup of $\operatorname{Gal}(L / K)$ defined as

$$
D(\beta \mid \wp)=\{\sigma \in \operatorname{Gal}(L / K) \mid \sigma(\beta)=\beta\} .
$$

Again, we denote by $L_{\beta}$ and $K_{\wp}$ the completions of $L$ and $K$ with respect to the valuations $v_{\beta}$ and $v_{\wp}$ associated to the prime ideals $\beta$ and $\wp$.

The following result relates the Galois group of an extension of number fields to the Galois group of the extension of their completions.

Proposition 1.5.1. In the previous hypothesis, the extension $L_{\beta} / K_{\wp}$ is normal and $\operatorname{Gal}\left(L_{\beta} / K_{\wp}\right)$ is isomorphic to the decomposition group $D(\beta \mid \wp)$.

Proof. The result comes from the fact that the decomposition group $D(\beta \mid \wp)$ consists precisely of those automorphisms which are continuous with respect to the valuation $v_{\beta}$. See for instance Neukirch's book [10], Ch. 2, $\S 9$, Proposition 9.6 .

We now give the definition of ramification groups, which shall be used in the study of the wild part of a Galois extension of $p$-adic fields.

Definition 1.11. Let $L / K$ be a Galois extension of $p$-adic fields and let $G=$ $\operatorname{Gal}(L / K)$. We define for $i \in \mathbb{N}$ the $i$-th ramification group of $L / K$ as

$$
G_{i}:=\left\{\sigma \in G \mid v_{\beta}(\sigma(x)-x) \geq i+1 \forall x \in R_{L}\right\} .
$$

The group $G_{0}$ is called the inertia group of $L / K$.

We now list some properties of these groups whose proof is an easy check.

Proposition 1.5.2. For the groups $G_{i}$ 's the following properties hold:

1. $G_{i} \triangleleft G$;

2. $G_{i}=1$ for some index $i$;

3. $G_{i} / G_{i+1}$ is a p-elementary abelian group for $i \geq 1$.

We end this section with a theorem which gives a complete description of the structure of the Galois group of an extension of $p$-adic fields.

Theorem 1.5.3. Let $L / K$ be a Galois extension of $p$-adic fields with Galois group $G$. Let $K^{\text {ur }}$ be the maximal unramified extension of $K$ contained in $L$ and $K^{\text {tame }}$ the maximal tame extension of $K$ in $L$. Suppose moreover that $e_{L / K}=e_{0} p^{s}$ with $p$ not diving $e_{0}$. Then we have: 
1. $\operatorname{Gal}\left(K^{u r} / K\right)=G / G_{0}$ is a cyclic group of order $f_{L / K}$;

2. $\operatorname{Gal}\left(K^{\text {tame }} / K^{u r}\right)=G_{0} / G_{1}$ and it is a cyclic group of order $e_{0}$;

3. $\operatorname{Gal}\left(L / K^{\text {tame }}\right)=G_{1}$ and its order is $p^{s}$;

4. $\operatorname{Gal}\left(L / K^{u r}\right)=G_{0}$, it has order $e_{L / K}$ and it is the semidirect product of a cyclic group of order prime to $p$ and a normal p-group.

Proof. See [9], Ch. 5, §2, Thm. 5.12.

The situation can be described using the following diagram:

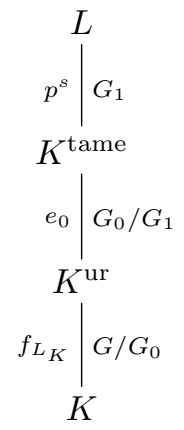

\subsection{Shafarevich's theorem on $p$-extensions}

We end this chapter with an important result of Shafarevich (see [13]) on $p$-extensions of $p$-adic fields, namely finite extensions whose degree is a power of $p$.

Shafarevich's theorem asserts that, although the Galois group of a p-extension is in general quite mysterious, there is a bound on the number of its generators. This result will become extremely useful in the ensuing chapters, when we will look for explicit bounds for the local degrees of an algebraic extension of $\mathbb{Q}$, since it permits, in some special situations, to bound the wild part of a $p$-adic extension.

Before we state Shafarevich's theorem we recall some basic results from local class field theory which shall be largely used in the proof of the theorem and in the next chapters. For further details on local class field theory we refer to Vostokov and Fesenko's book [4], Ch. IV.

Local class field theory establishes a one-to-one correspondence between finite abelian extensions of a $p$-adic field $K$ and open subgroups of finite index of $K^{*}$. This correspondence is such that if $L / K$ is a finite abelian extension then it corresponds to the open subgroup $N_{L / K}\left(L^{*}\right)$ of $K^{*}$, where $N_{L / K}$ is the norm function of the extension $L / K$. In addition we have

$$
\operatorname{Gal}(L / K) \simeq K^{*} / N_{L / K}\left(L^{*}\right) .
$$

We recall the following definitions of exponent of a group and exponent of a Galois extension. 
Definition 1.12. A group $G$ has exponent $b$ if $b$ is the smallest positive integer such that $g^{b}=1$ for every $g \in G$ and we denote it by $\exp (G)$.

We say that a Galois extension has $L / K$ exponent $b$ if $\exp (\operatorname{Gal}(L / K))=b$.

We have the following proposition.

Proposition 1.6.1. Let $K$ be a $p$-adic field and let $L / K$ be the maximal abelian extension of $K$ of exponent $b$. Then

$$
\operatorname{Gal}(L / K) \simeq K^{*} /\left(K^{*}\right)^{b} .
$$

Proof. From local class field theory, the extension $L$ corresponds to the smallest open subgroup $H$ of $K^{*}$ of finite index such that $K^{*} / H$ has exponent $b$.

Now if $H$ is a subgroup of $K^{*}$ and $\exp \left(K^{*} / H\right)=b$, then $\left(K^{*}\right)^{b} \leq H$ and $\left(K^{*}\right)^{b}$ is the smallest open subgroup of $K^{*}$ with the required property.

We can now state and prove Shafarevich's theorem.

Theorem 1.6.2 (Shafarevich). Let $p$ be an odd prime, $K$ a $p$-adic field of degree $n$ over $\mathbb{Q}_{p}$ and $L / K$ a Galois p-extension with Galois group $H$. Then $H$ has at most $n+2$ generators.

Proof. We notice that the number of generators of $H$ equals the number of generators of $H / \Phi(H)$, where $\Phi(H)$ is the Frattini subgroup of $H$, namely the intersection of $H$ and all proper maximal subgroups of $H$. Since $H$ is a $p$-group, it is well known that $\Phi(H)$ is the smallest normal subgroup of $H$ such that the quotient group $H / \Phi(H)$ is an elementary abelian group.

We have that the extension $L^{\Phi(H)} / K$ has Galois group isomorphic to $H / \Phi(H)$, therefore it is contained in the compositum, say $F$, of all $p$-elementary abelian extensions of $K$. From local class field theory we know that there is a correspondence between finite abelian extensions of $K$ and subgroups of $K^{*}$ of finite index. Now the group $K^{*}$ is isomorphic to

$$
K^{*} \simeq \mathbb{Z} \oplus \mathbb{Z} /(q-1) \mathbb{Z} \oplus \mathbb{Z} / p^{a} \mathbb{Z} \oplus \mathbb{Z}_{p}^{\left[K: \mathbb{Q}_{p}\right]}
$$

where $q$ is the order of the residue field $\kappa_{K}$ and $a \geq 0$ is the maximal integer with the property that $K$ contains a primitive $p^{a}$-th root of unity (see [9], Ch. V, $§ 1$, Prop. 5.8 and Thm. 5.7). Then, in view of Proposition 1.6.1, we have

$$
\operatorname{Gal}(F / K)=\left(K^{*}\right) /\left(K^{*}\right)^{p} \simeq(\mathbb{Z} / p \mathbb{Z})^{\left[K: \mathbb{Q}_{p}\right]+\epsilon(K)}
$$

where $\epsilon(K)=1$ if $K$ does not contain $p$-th roots of unity and $\epsilon(K)=2$ otherwise. Therefore $H / \Phi(H)$ has at most $n+2$ generators, and this proves the result.

Remark 1.5. Actually, Shafarevich's result is stronger and it asserts that a $p$-group $H$ can occur as the Galois group of an extension of a $p$-adic field $K$ not containing $p$-th roots of unity and such that $\left[K: \mathbb{Q}_{p}\right]=n$ if and only if $H$ has at most $n+1$ generators. We will omit the whole proof of this result since it is quite long and we will only need the implication proved above. We refer the interested reader to Shafarevich's paper [13]. 


\section{Chapter 2}

\section{Galois extensions with finite exponents and the abelian case}

In this work we shall mainly investigate the mutual relations of three properties of an infinite algebraic extension of $\mathbb{Q}$ : having uniformly bounded local degrees; being contained in the compositum of a family of number fields of bounded degree; being generated by elements of bounded degree. In particular we could ask whether these three properties are equivalent.

This chapter has three purposes. The first is to study some properties of the compositum of fields with bounded degrees, in order to give some motivations to our questions, and this will be done in Section 2.1. The second is to give a characterization of Galois extensions with uniformly bounded local degrees: we shall prove in Section 2.2 that for an infinite Galois extension of $\mathbb{Q}$ having uniformly bounded local degrees at almost every prime is equivalent to have uniformly bounded local degrees at every prime and that extensions with these properties are exactly those whose Galois group has finite exponent. The first part of the proof is an easy application of Chebotarev's density theorem; the second part is based on a result of Shafarevich on $p$-extensions plus the work of Zelmanov on the restricted Burnside problem. We also provide, in Section 2.3 , explicit bounds for the local degrees of a Galois extension $K$ of $\mathbb{Q}$ of finite exponent $b$ in the special case when there is a bound on the order of the wildly ramified part of the Galois group of the completion of every finite Galois subextension of $K$. The last goal is to give a positive answer to our initial question in the case where $K$ is an abelian extension of $\mathbb{Q}$ and we shall prove this in Section 2.4 .

Throughout this work we denote by $\mathbb{Q}^{\text {alg }}$ a fixed algebraic closure of $\mathbb{Q}$. 


\subsection{Definitions and motivations}

We let $d$ be a positive integer and $F$ a number field. We denote by $F^{(d)}$ the field obtained by taking the compositum of all algebraic extensions of $F$ of degree at most $d$ (over $F$ ). One of the questions posed in this work is whether every algebraic extension of $\mathbb{Q}$ with uniformly bounded local degrees is contained in $\mathbb{Q}^{(d)}$ for some positive integer $d$. In order to understand this question, we need to explain what does it mean for an infinite algebraic extension $K$ of $\mathbb{Q}$ to have uniformly bounded local degrees. We fix some notation. For every prime number $p$ we define a valuation $v_{p}$ of $K$ which extends the $p$-adic valuation over $\mathbb{Q}$ in the following way. We fix an embedding $\sigma: K \hookrightarrow \mathbb{C}_{p}$, where $\mathbb{C}_{p}$ is the $p$-adic completion of $\mathbb{Q}_{p}^{\text {alg }}$. By Corollary 1.2 .5 , there is a unique valuation $w_{p}$ of $\mathbb{C}_{p}$ which extends the $p$-adic valuation of $\mathbb{Q}$. Then we define the valuation $v_{p}$ of $K$ as $v_{p}(x):=w_{p}(\sigma(x))$ for every $x \in K$.

The completion of $K$ with respect to $v_{p}$ is denoted by $K_{v_{p}}$ and it is an algebraic extension of $\mathbb{Q}_{p}$. This extension in general is not finite, but sometimes it is. We are interested in the case when this extension is finite and moreover its degree is bounded, for every prime $p$ and every valuation $v_{p}$ above $p$, by a constant independent from $p$. We have the following definition.

Definition 2.1. Let $K$ be an algebraic extension of $\mathbb{Q}$. Then $K$ has uniformly bounded local degrees if there exists a positive integer $B$ such that $\left[K_{v_{p}}: \mathbb{Q}_{p}\right]<B$ for every prime number $p$ and every valuation $v_{p}$ of $K$ extending the $p$-adic valuation of $\mathbb{Q}$.

We say that $K$ has uniformly bounded local degrees at almost every prime if the set of primes for which the local degrees are not uniformly bounded has finite cardinality.

Our initial question, roughly speaking, is whether the fields $\mathbb{Q}^{(d)}$ are maximal among those all with uniformly bounded local degrees. In order to give a motivation to this we have to show that the fields $\mathbb{Q}^{(d)}$ have uniformly bounded local degrees. This result has been already proved in paper [2] and in Bombieri and Gubler's book [1], which largely study the field $\mathbb{Q}^{(d)}$ in relation to the Northcott property. We now want to recall the proof of this fact; in order to do this we need the following lemma.

Lemma 2.1.1. Let $L / \mathbb{Q}_{p}$ be a finite extension of degree $n_{0}$ and fix a positive integer $n$. Then the number of extensions of $L$ of degree $n$ is finite and bounded by a constant which does not depend on $p$.

Proof. From Corollary 1.4.2, we know that every extension of $L$ of degree $n$ is given by an unramified subextension of degree $f$ and a totally ramified extension of degree $e=n / f$, for every $f$ dividing $n$. We notice that we have a finite number, independent of $p$, of choices for $f$ and $e$.

For every choice of $f$, we denote by $M_{f}$ the unique unramified extension of degree $f$ of $L$ and we set $d:=\left[M_{f}: \mathbb{Q}_{p}\right]=f n_{0}$. So we are left to prove that any $p$-adic field has only a finite number, independent of $p$, of totally ramified extensions of fixed degree $e$. 
We shall now use a formula obtained by Krasner in [6] which computes the number $\mathcal{R}_{p, e}(d)$ of totally ramified extensions of degree $e$ of a $p$-adic field of degree $d$ over $\mathbb{Q}_{p}$.

Setting $N:=e d$ and writing $e=p^{m} h$, with $(h, p)=1$, we have:

$$
\mathcal{R}_{p, e}(d)=e \sum_{s=0}^{m} p^{s}\left(p^{\epsilon(s) N}-p^{\epsilon(s-1) N}\right)
$$

where

$$
\epsilon(s):=\frac{1}{p}+\frac{1}{p^{2}}+\ldots+\frac{1}{p^{s}}
$$

for any integer $s$ greater than $0, \epsilon(0):=0$ and $\epsilon(-1):=-\infty$.

If we fix $e$ and we let $p$ varies, the number of $p$ for which $m$ is nonzero is finite (if $m=0$ we are considering totally ramified and tame extensions of degree $e$ of a $p$-adic field and this number is known to be $e$ ).

We define two numbers $R_{e}(d):=\max _{p \mid e} \mathcal{R}_{p, e}(d)$ and $B_{e}(d):=\max \left(e, R_{e}(d)\right)$. We have that, for every prime $p$, a $p$-adic field of degree $d$ over $\mathbb{Q}_{p}$ has at most $B_{e}(d)$ totally ramified extensions of degree $e$ and this bound does not depend on $p$. Then $B:=\max _{e \mid n} B_{e}\left((n / e) n_{0}\right)$ is a bound on the number of extensions of degree $n$ of a $p$-adic field of degree $n_{0}$ over $\mathbb{Q}_{p}$ and it is independent from $p$.

Remark 2.1. Finiteness for the number of extensions of $\mathbb{Q}_{p}$ can be proved just using some compactness arguments and Krasner's lemma (Lemma 1.3.2). In fact, from Proposition 1.4.5, every totally ramified extension of degree $e$ of a $p$-adic field $K$ is generated by the roots of an Eisenstein's polynomial of degree $e$. The space of Eisenstein's polynomials is isomorphic to the space

$$
\underbrace{\wp \times \wp \times \ldots \times \wp \times\left(\wp^{2}-\wp\right)}_{e \text { times }}
$$

which is the space of coefficients of such polynomials, where $\wp$ is the maximal ideal of the valuation ring $R$ of $K$.

We notice that this space is compact, being a product of compact spaces $(\wp$ is a closed subset of $R$ which is compact, see [9], Ch. 5, $\S 1$, Thm. 5.2). So every covering of this space admits a finite subcovering.

In view of Corollary 1.3.3, if two polynomials of the same degrees have their coefficients close enough, then their roots will generate the same extension of $p$-adic fields. Hence the number of extensions generated by the roots of all Eisenstein's polynomials of degree $e$ is finite and so is the number of totally ramified extensions of degree $e$ of a $p$-adic field.

Now we are able to prove the following proposition.

Proposition 2.1.2. Let $F$ be a number field of degree $n$ over $\mathbb{Q}$ and let $v$ be any place of $F$. Let $w$ be an extension of $v$ to $F^{(d)}$ and denote by $F_{w}^{(d)}$ its completion. Then the local degree $\left[F_{w}^{(d)}: F_{v}\right]$ is bounded solely in terms of $n$ and $d$. 
Proof. Denoting by $\left\{F_{i}\right\}_{i}$ the family of all extensions of $F_{i} / F$ such that $\left[F_{i}\right.$ : $F] \leq d$, we have that the field $F^{(d)}$ can be obtained as the compositum of this family.

Since $F_{v}$ is a $p$-adic field, for some prime $p$, by Lemma 2.1 .1 , it has only a finite number, say $r$, of extensions of degree at most $d$ and this number depends only on $d$ and $\left[F_{v}: \mathbb{Q}_{p}\right] \leq n$. Let us denote by $\mathcal{S}=\left\{F_{v, 1}, \ldots, F_{v, r}\right\}$ the set of these extensions and by $F_{v}^{\prime}$ their compositum. We notice that the degree $\left[F_{v}^{\prime}: F_{v}\right]$ is bounded solely in terms of $d$ and $n$.

Denote by $F_{i, w}$ the completion of $F_{i}$ at the valuation induced by the restriction of $w$ to $F_{i}$. Then $F_{i, w}$ is an extension of $F_{v}$ of degree at most $d$ and thus it belongs to $\mathcal{S}$ for every $i$. Therefore $F_{w}^{(d)} \subseteq F_{v}^{\prime}$ and this concludes the proof.

\subsection{Characterization of fields with uniformly bounded local degrees}

In this section we give a characterization of Galois extensions with uniformly bounded local degrees at almost every prime only in terms of the exponent of their Galois groups. In particular we want to prove that an infinite Galois extension $K$ of $\mathbb{Q}$ has uniformly bounded local degrees if and only if the group $\operatorname{Gal}(K / \mathbb{Q})$ has finite exponent.

Remark 2.2. It can be easily checked that, if $F$ is a number field, then $F^{(d)} / F$ is a Galois extension with Galois group of finite exponent.

In fact, for every extension $L / F$ of degree at most $d$ with $L=F(\alpha)$, the conjugates of $\alpha$ in $F^{\text {alg }}$ also generate extensions of degree at most $d$. Therefore the Galois closure of $L$ is a subfield of $F^{(d)}$ which is a Galois extension, being the compositum of a family of Galois extensions.

Now for every extension $L / F$ with $[L: F] \leq d$, we denote by $L^{\prime}$ the normal closure of $L$ in the algebraic closure of $F$. We have that $\left[L^{\prime}: F\right] \leq d$ ! and therefore $\exp \left(\operatorname{Gal}\left(L^{\prime} / F\right)\right)$ divides $d$ !. Clearly the compositum of all the extensions $L^{\prime}$ 's has also Galois group of exponent dividing $d$ ! and contains $F^{(d)}$.

Although we assume the reader to be familiar with finite and infinite Galois theory, we summarize some facts which will be used. We recall that if $K / \mathbb{Q}$ is an infinite algebraic Galois extension then its Galois group $\operatorname{Gal}(K / \mathbb{Q})$ has a structure of profinite group: this is obtained constructing it as the inverse limit of the projective system of groups $\{\operatorname{Gal}(E / \mathbb{Q})\}_{E}$, where $E$ varies among all the finite Galois extensions of $\mathbb{Q}$ contained in $K$, the inverse limit being taken under the partial order given by the inclusion relation.

We also recall, for finite Galois extensions, the Chebotarev's Density Theorem.

Theorem 2.2.1 (Chebotarev's Density Theorem). Let $L / K$ be a Galois extension of number fields of degree $n$ and let $A$ be an arbitrary class of conjugate elements in the Galois group $\operatorname{Gal}(L / K)$. For every prime $\wp$ of $K$ unramified in $L / K$ denote by $F_{L / K}(\wp)$ the conjugacy class in $\operatorname{Gal}(L / K)$ of the Frobenius 
automorphism (generating the decomposition group) associated with any prime lying above $\wp$. Then the set

$$
P_{A}:=\left\{\wp \mid F_{L / K}(\wp)=A\right\}
$$

is infinite and has Dirichlet density $|A| / n$.

Proof. See [9], Ch.7, §3, Thm. 7.10.

We now want to prove the following theorem.

Theorem 2.2.2. Let $L$ be a number field and $K / L$ an infinite algebraic Galois extension of $L$. Then the following conditions are equivalent:

(1) $K$ has uniformly bounded local degrees at every prime of $L$;

(2) $K$ has uniformly bounded local degrees at almost every prime of $L$;

(3) $\operatorname{Gal}(K / L)$ has finite exponent.

Proof. It is trivial to show that (1) implies (2). Now we suppose that (2) holds, that is $K$ has local degrees uniformly bounded by a constant $B$ at every prime except some primes belonging to a finite set $S$. We fix a finite Galois extension $E$ of $L$ contained in $K$ and we take $\sigma \in \operatorname{Gal}(E / L)$. By Chebotarev's Density Theorem there exist a prime $\wp$ of $L$ with $\wp \notin S$, a prime $\beta$ of $E$ unramified above $\wp$ and a conjugate $\tau$ of $\sigma$ that generates the decomposition group $D(\beta \mid \wp)$ which is cyclic and isomorphic to $\operatorname{Gal}\left(E_{\beta} / L_{\wp}\right)$, where $E_{\beta}$ and $L_{\wp}$ denote the completions of $E$ and $L$ with respect to $\beta$ and $\wp$ respectively. By assumption, $\left|\operatorname{Gal}\left(E_{\beta} / L_{\wp}\right)\right| \leq B$, thus $\sigma^{B !}=\tau^{B !}=$ id and $\exp (\operatorname{Gal}(E / L)) \leq B$ !. Since $\operatorname{Gal}(K / L)$ is the inverse limit of the family $\{\operatorname{Gal}(E / L)\}_{E}$, where $E$ varies among the finite Galois extension of $L$ contained in $K$, we have $\exp (G) \leq B$ ! and (3) holds.

Now we suppose that (3) holds for $K$ and we set $\exp (\operatorname{Gal}(K / L))=b$. We want to prove that the local degrees of $K$ are uniformly bounded at every primes.

We fix a prime number $p$ and we consider a valuation $w$ of $K$ above $p$. We write $K$ as a compositum of a family of number fields $\left\{K_{m}\right\}_{m}$, where $K_{m} / L$ is any finite Galois extension of $L$ contained in $K$ with Galois group $G_{m}=$ $\operatorname{Gal}\left(K_{m} / L\right)$.

We notice that $v=\left.w\right|_{K_{m}}$ is a valuation of $K_{m}$ above $p$ and we denote by $K_{m, v}$ and $L_{v}$ the completions of $K_{m}$ and $L$ with respect to $v$ and $\left.v\right|_{L}$. We recall that, by Proposition 1.5.1, $\operatorname{Gal}\left(K_{m, v} / L_{v}\right)$ is isomorphic to a subgroup of $G_{m}$ and therefore it has exponent at most $b$. We have three cases for the extension $K_{m, v} / L_{v}$ :

(i) if it is unramified, then it is cyclic of order bounded by $b$;

(ii) if it is tamely ramified, then it is a metacyclic extension, thus its order is bounded by $b^{2}$; 
(iii) if it is wildly ramified, then the tamely ramified part has always degree at most $b^{2}$. We know that the first ramification group of $K_{m, v} / L_{v}$ is a $p$-group of exponent at most $b$ and Theorem 1.6.2 tells us that it has at most $n b^{2}+2$ generators, where $n=\left[L: \mathbb{Q}_{p}\right]$ and $n b^{2}$ is a bound for the degree over $\mathbb{Q}_{p}$ of the tamely ramified part of the extension.

So the problem reduces to the following: is it true that, for every positive integers $b$ and $n$, if a finite group $H$ has $n$ generators and exponent $b$, then the order of $H$ is bounded by a constant which depends only on $n$ and $b$ ?

This question is known as the Restricted Burnside's Problem and was positively answered in 1989 by Efim Zelmanov; Zelmanov's proof is very difficult and it is definitely beyond the intent of this work; for a detailed description of Zelmanov's proof the interested reader should refer to Vaughan-Lee's book [15].

In view of Zelmanov's positive answer to the Restricted Burnside's Problem, the wildly ramified part of the extension has a degree which is bounded by a constant depending only on $n$ and $b$.

We can now conclude our proof. Summing up all the previous results, we get that for every $m$, the local degree $\left[K_{m, v}: \mathbb{Q}_{p}\right]$ is bounded by a constant depending only on $b$ and $n=[L: \mathbb{Q}]$, which are fixed. Thus, by Lemma 2.1.1, the number of all possible completions of the $K_{m}$ 's at primes above $p$ is finite and independent from $p$. Moreover the compositum of this finite number of fields has degree over $\mathbb{Q}_{p}$ which is bounded by a constant independent of $p$ and it contains the completion of $K$ with respect to every valuation extending the $p$-adic one.

\subsection{A formula for bounding the local degrees}

The proof of the previous result does not provide an explicit bound for the local degrees of an extension $K / \mathbb{Q}$ of finite exponent. In order to compute effectively such a bound, we need additional informations on the order of the wild part of the completion of every finite Galois subextension of $K$. We have the following result.

Theorem 2.3.1. Let $K / \mathbb{Q}$ be an infinite Galois extension of exponent $b$ and let $\left\{K_{m}\right\}_{m}$ be the family of all finite Galois subextensions of $K$. Suppose that for every $m$, every prime number $p$ dividing $b$ and every valuation $v$ of $K_{m}$ above $p$ we have $v_{p}\left(\left|\operatorname{Gal}\left(K_{m, v} / \mathbb{Q}_{p}\right)\right|\right) \leq n$, where $v_{p}$ denotes the usual $p$-adic valuation of $\mathbb{Q}$.

Then $K$ has local degrees bounded by

$$
\prod_{i=1}^{n} A(i)
$$

where $A(1)=b^{3}$ and

$$
A(i+1)=b^{\left(\prod_{j=1}^{i} A(j)\right)+2} .
$$


Proof. We fix a prime number $p$ and we consider a valuation $w$ of $K$ extending the $p$-adic valuation of $\mathbb{Q}$. The field $K$ equals the compositum of all its finite Galois subextensions $\left\{K_{m}\right\}_{m}$ and, for every $m, v=\left.w\right|_{K_{m}}$ is a valuation of $K_{m}$ above $p$. We denote by $K_{w}$ and by $K_{m, v}$ the completions of $K$ and $K_{m}$ with respect to $w$ and $v$, respectively.

We fix $m$ and we set $G=\operatorname{Gal}\left(K_{m, v} / \mathbb{Q}_{p}\right)$. We consider the filtration of $G$ via its ramification subgroups

$$
G \triangleright G_{0} \triangleright G_{1} \triangleright G_{2} \triangleright \ldots \triangleright G_{r}=1
$$

where:

- $G / G_{0}$ is cyclic being the Galois group of the unramified part of the extension $K_{m, v} / \mathbb{Q}_{p}$;

- $G_{0} / G_{1}$ is the Galois group of the tame and totally ramified part of the extension and it is also cyclic;

- $G_{i} / G_{i+1}$ is $p$-elementary abelian for every $i \geq 1$.

If $p$ does not divide the exponent $b$, then the local extension can only be tamely ramified. In this case the unramified part of the extension $K_{m, v}^{G_{0}} / \mathbb{Q}_{p}$ is contained in the compositum $L^{\mathrm{ur}, b}$ of all unramified extensions of $\mathbb{Q}_{p}$ of degree dividing $b$, which has degree $\left[L^{\mathrm{ur}, b}: \mathbb{Q}_{p}\right]=b$, since we have only one unramified extension of $\mathbb{Q}_{p}$ of every fixed degree. Therefore the maximal unramified subextension $K_{w}^{\mathrm{ur}}$ of $K_{w}$ is also contained in $L^{\mathrm{ur}, b}$.

The tame and totally ramified part of the extension $K_{m, v}^{G_{1}} / K_{m, v}^{G_{0}}$ is contained in the maximal abelian extension $L^{\text {tame,b}}$ of $L^{\mathrm{ur}, b}$ of exponent dividing $b$ and prime to $p$. From Proposition 1.6.1 we have

$$
\operatorname{Gal}\left(L^{\mathrm{tame}, b} / L^{\mathrm{ur}, b}\right) \simeq L^{\mathrm{ur}, b^{*}} /\left(L^{\mathrm{ur}, b^{*}}\right)^{a}
$$

for some integer $a$ prime to $p$ and smaller than $b$. Therefore

$$
\left|\operatorname{Gal}\left(L^{\text {tame }, b} / L^{\mathrm{ur}, b}\right)\right| \leq|(\mathbb{Z} / a \mathbb{Z})|^{2} \leq b^{2} .
$$

Since this holds for every $m$, the maximal tame subextension $K_{w}^{\text {tame }}$ of $K_{w}$ is a subfield of $L^{\text {tame, } b}$.

If $p$ divides the exponent $b$, then one can have wild ramification. However, using the previous arguments, the degree over $\mathbb{Q}_{p}$ of the maximal tame subextension $K_{w}^{\text {tame }}$ of $K_{w}$ is bounded by $b^{3}$.

As for the wildly ramified part of the extension, since by hypothesis $v_{p}(|G|) \leq$ $n$, the group $G_{1}$ has order at most $p^{n}$ and the filtration

$$
G_{1} \triangleright G_{2} \triangleright \ldots \triangleright G_{r}=1
$$

has at most $n$ jumps; this means that $G_{i} \neq G_{i+1}$ for at most $n$ indexes $i$.

Therefore we have the following tower of fields

$$
\mathbb{Q}_{p}=K_{m, v}^{G} \subseteq K_{m, v}^{G_{0}} \subseteq K_{m, v}^{G_{1}} \subseteq \ldots \subseteq K_{m, v}^{G_{r}}=K_{m, v}
$$


in which the extension $K_{m, v}^{G_{i+1}} / K_{m, v}^{G_{i}}$ is elementary $p$-abelian for at most $n$ indexes $i \geq 1$ and trivial otherwise.

We set $L_{1}=L^{\text {tame,b}}$ and we denote by $L_{i+1}$ the compositum of all $p$ elementary abelian extensions of $L_{i}$. We notice that $K_{m, v}^{G_{j}}$ is contained in some $L_{n}$ for every index $j$. Again from Proposition 1.6.1, we have that

$$
\operatorname{Gal}\left(L_{i+1} / L_{i}\right)=\left(L_{i}^{*}\right) /\left(L_{i}^{*}\right)^{p} \simeq(\mathbb{Z} / p \mathbb{Z})^{\left[L_{i}: \mathbb{Q}_{p}\right]+\epsilon\left(L_{i}\right)}
$$

where $\epsilon\left(L_{i}\right)=1$ if $L_{i}$ does not contain $p$-th roots of unity and $\epsilon\left(L_{i}\right)=2$ otherwise. Since $p<b$ and $\left[L_{1}: \mathbb{Q}_{p}\right] \leq b^{3}=A(1)$, we have $\left[L_{2}: L_{1}\right] \leq b^{b^{3}+2}=$ $A(2)$ and, using formula 2.2 , it is easy to see that $\left[L_{i}: L_{i-1}\right] \leq A(i)$. Since $K_{w}$ is contained in $L_{n}$, the result follows.

\subsection{The abelian case}

We consider an infinite abelian extension $K$ of $\mathbb{Q}$, namely an infinite algebraic normal extension $K$ of $\mathbb{Q}$ with abelian profinite Galois group. The aim of this section is to prove the following theorem.

Theorem 2.4.1. For an infinite abelian extension $K$ of $\mathbb{Q}$ the following conditions are equivalent:

(a) K has uniformly bounded local degrees;

(b) there exists a positive integer d such that $K$ is contained in $\mathbb{Q}^{(d)}$;

(c) every finite abelian subextension of $K$ can be generated by elements of bounded degree.

Proof. It is trivial to prove that (c) implies (b). The fact that (b) implies (a) follows from Proposition 2.1.2.

We now assume that $K$ satisfies condition (a). We set $G=\operatorname{Gal}(K / \mathbb{Q})$; then, by Theorem $2.2 .2, \exp (G) \leq b$ for some positive integer $b$ and

$$
G={\underset{m}{\lim }}_{\lim _{m}}
$$

where $G_{m}=\operatorname{Gal}\left(K_{m} / \mathbb{Q}\right)$ and $K_{m}$ is any finite abelian extensions of $\mathbb{Q}$ contained in $K$. Since $G_{m}$ is a finite abelian group, we can write it as a product of finite cyclic groups

$$
G_{m}=\prod_{i=1}^{n} U_{i} .
$$

We let $H_{i}$ be the subgroup of $G_{m}$ defined as

$$
H_{i}:=\prod_{j \neq i} U_{j} .
$$

We have $\left(G_{m}: H_{i}\right)=\left|U_{i}\right|=\exp \left(U_{i}\right) \leq b$ for all $i$ 's and $\cap_{i=1}^{n} H_{i}=1$. 
Therefore $K_{m}$ is the compositum of its subextensions $\left\{K_{m}^{H_{i}}\right\}_{i}$ and these extensions satisfy the inequality $\left[K_{m}^{H_{i}}: \mathbb{Q}\right]=\left(G_{m}: H_{i}\right) \leq b$. Thus $K_{m}$ is generated by elements of bounded degree and $K$ satisfies condition (c), which completes the proof.

Remark 2.3. We notice that, from local class field theory, an abelian extension of exponent $b$ has local degrees bounded by $b^{3}$.

In fact if $K / \mathbb{Q}$ is an abelian extension of exponent $b$, the completion of $K$ with respect to a prime $p$ is contained in the maximal abelian extension $M$ of $\mathbb{Q}_{p}$ of exponent $b$; in view of Theorem 1.6.1 we have that

$$
\left|\operatorname{Gal}\left(M / \mathbb{Q}_{p}\right)\right|=\left|\mathbb{Q}_{p}{ }^{*} /\left(\mathbb{Q}_{p}{ }^{*}\right)^{b}\right| \leq b^{3} .
$$

Actually, we have proved a slightly more general result, which follows as an easy corollary.

Corollary 2.4.2. Let $K / \mathbb{Q}$ be a field extension with uniformly bounded local degrees and suppose that the extension $K / L$ is abelian for some number field $L \subset K$. Then there exists a positive integer $d$ such that $K \subset \mathbb{Q}^{(d)}$.

Proof. We notice that $K / L$ has also uniformly bounded local degrees. Proposition 2.4 shows that $K$ is contained in $L^{\left(d^{\prime}\right)}$ for some integer $d^{\prime}$. If $n=[L: \mathbb{Q}]$ and we set $d=d^{\prime} n$, then $K$ is also contained in $\mathbb{Q}^{(d)}$.

Remark 2.4. It is immediate to see that Corollary 2.4 .2 does not provide a characterization of all extensions of $\mathbb{Q}$ contained in some $\mathbb{Q}^{(d)}$. This would be the same as saying that, for every fixed $d$ and for every subfield $K$ of $\mathbb{Q}^{(d)}$, there exists a number field $L$ with $\mathbb{Q} \subseteq L \subset K$ such that $K / L$ is a Galois and abelian extension.

This is true for $d=2$, since in this case $\mathbb{Q}^{(2)} / \mathbb{Q}$ is abelian, but it is easily seen to be false in general. In fact if we consider an infinite family $\left\{K_{i}\right\}_{i}$ of linearly disjoint Galois extensions of $\mathbb{Q}$ with $\operatorname{Gal}\left(K_{i} / \mathbb{Q}\right)=S_{3}$ for every $i$ (the existence of such a family is provided, for instance, in [12], §3.3, Example 1 and $\S 4.1$, Example 2). Then the field $K$ obtained by taking the compositum of the family is contained in $\mathbb{Q}^{(6)}$ and it has Galois group $\operatorname{Gal}(K / \mathbb{Q})=\prod_{i=1}^{\infty} G_{i}$ with $G_{i} \simeq S_{3}$ for all $i$ 's. However, there is no number field $L \subset K$ such that $\operatorname{Gal}(K / L)$ is abelian, otherwise $G$ would have abelian subgroups of finite index, which is not possible. 


\section{Chapter 3}

\section{Extraspecial groups}

This chapter introduces definitions and properties of extraspecial groups, namely $p$-groups in which the center and the commutator subgroup are both cyclic of order $p$.

The fundamental relevance of these groups in this work will become evident in the following chapters, when we will prove the existence both of fields with uniformly bounded local degrees not contained in $\mathbb{Q}^{(d)}$ for any positive integer $d$ and of subfields of $\mathbb{Q}^{(d)}$ which cannot be generated by elements of bounded degree. In order to do this we need to prove the following properties of extraspecial groups:

(i) for every odd prime $p$ and every positive integer $m$ there exists an extraspecial group $G_{m}$ of order $p^{2 m+1}$ and exponent $p$ and it can be obtained as a quotient of the direct product $E_{1} \times \ldots \times E_{m}$, where $E_{1}, \ldots, E_{m}$ are extraspecial groups of order $p^{3}$ and exponent $p$;

(ii) every group $G_{m}$ has an irreducible module over some finite field of dimension $p^{m}$

(iii) every subgroup of $G_{m}$ of index at most $p^{m}$ contains the center of $G_{m}$.

Property (i) is proved in Sections 3.1 and 3.2. In Section 3.1 we give some definitions and we discuss the basic properties of extraspecial groups. In Section 3.2 we will introduce a special central product of groups called amalgamated central product and we will show that an extraspecial group of order $p^{2 m+1}$ and exponent $p$ can be obtained as an amalgamated central product of $m$ extraspecial groups of order $p^{3}$ and exponent $p$ and that this product satisfies property (i). We will also give, for completeness, the structure theorem for extraspecial groups. This theorem asserts that every extraspecial group of order $p^{2 m+1}$ and exponent $p$ can be obtained as in (i) and that, moreover, if $p$ is odd, such a group is unique up to isomorphisms.

Property (ii) is proved in Section 3.3, where an explicit construction of an irreducible module over certain finite fields is made. This will be used to con- 
struct a field with uniformly bounded local degrees not contained in $\mathbb{Q}^{(d)}$ for any positive integer $d$.

Finally in Section 3.4 we prove property (iii), which will be used in the proof of the main theorem, in Chapter 4 , to provide an example of subfields of $\mathbb{Q}^{\left(p^{3}\right)}$ which cannot be generated by elements of bounded degree.

A detailed discussion on extraspecial groups and related topics can be found in Doerk and Hawkes' book [3].

\subsection{Definitions and first properties}

The purpose of this section is to define extraspecial groups and to describe their basic structure. This can be easily done viewing these groups as symplectic vector spaces over finite fields.

Definition 3.1. Let $p$ be a prime. A $p$-group $E$ is said to be extraspecial if the center $Z(E)$ and the commutator subgroup $E^{\prime}$ have order $p$.

An extraspecial group has different important properties. Before we state them, we recall a lemma which shall be used in the sequel.

Lemma 3.1.1. Let $G$ be a finite group. If $x, y \in G$ are such that $[x, y] \in Z(G)$, then $\left[x^{m}, y\right]=[x, y]^{m}$ for every $m \geq 1$.

Proof. The formula trivially holds for $m=1$. Now we suppose it is true for $l<m$. Then

$$
\left[x^{m}, y\right]=x^{m} y x^{-m} y^{-1}=x^{m-1}[x, y]\left(y x x^{-m} y^{-1}\right)=[x, y]\left[x^{m-1}, y\right]
$$

and we conclude.

We are now able to prove the following proposition.

Proposition 3.1.2. For an extraspecial p-group $E$ the following properties hold:

1. $Z(E)=E^{\prime}=\Phi(E)$, where $\Phi(E)$ is the Frattini subgroup of $E$;

2. the quotient $E / Z(E)$ is elementary abelian;

3. if $p \neq 2$, for every $x, y \in E$ we have $(x y)^{i}=x^{i} y^{i}[y, x]^{\frac{i(i-1)}{2}}$ and $(x y)^{p}=$ $x^{p} y^{p}$.

Proof. 1. Since $E$ is a $p$-group and $E^{\prime}$ is a normal subgroup of $E$, the intersection $E^{\prime} \cap Z(E)$ is nontrivial, hence $Z(E)=E^{\prime}$ since they are both cyclic of prime order. Now, by the Burnside basis theorem (see [3], Ch. A, $\S 9$, Thm. 9.6), we have that $\Phi(E)=E^{p} E^{\prime}=E^{p} Z(E)$. If $g \in E$, then, using Lemma 3.1.1, we get $\left[g^{p}, h\right]=[g, h]^{p}=1$, hence $E^{p} \subseteq Z(E)$ and $\Phi(E)=Z(E)=E^{\prime}$. 
2. This follows immediately from the equality $Z(E)=\Phi(E)$ since $\Phi(E)$ is the smallest normal subgroup of $P$ such that the quotient $E / \Phi(E)$ is elementary abelian.

3. The formula is true for $i=1$ and we suppose it to be true for $l \leq i$. Then, using Lemma 3.1.1, we get

$$
(x y)^{i+1}=(x y)^{i}(x y)=x^{i} y^{i}[y, x]^{\frac{i(i-1)}{2}} x y=\left(x^{i}\left(y^{i} x\right) y\right)[y, x]^{\frac{i(i-1)}{2}} .
$$

and since $y^{i} x=[y, x]^{i} x y^{i}=x y^{i}[y, x]^{i}$ we have

$$
(x y)^{i+1}=x^{i+1} y^{i+1}[y, x]^{\frac{(i+1) i}{2}} .
$$

In particular, since $[y, x]^{p}=1$, for $i=p$ we have that $(x y)^{p}=x^{p} y^{p}$.

\subsubsection{Symplectic spaces and quadratic forms}

We want to recall some basic facts about symplectic spaces and hyperbolic planes.

Definition 3.2. Let $V$ be a finite dimensional vector space over a field $K$. Then $V$ is a symplectic space if $V$ is endowed with a bilinear form $f: V \times V \rightarrow K$ such that $f(v, v)=0$ for every $v \in V$. Such an $f$ is called a symplectic form on $V$.

If $U$ is a subspace of a symplectic space $V$ we define the orthogonal subspace of $U$ as

$$
U^{\perp}=\{w \in V \mid f(u, w)=0 \quad \forall u \in U\} .
$$

If $W \leq U^{\perp}$ we write $U \perp W$. $V$ is said to be non-degenerate if $V^{\perp}=0$.

We want to define isometries of symplectic spaces.

Definition 3.3. If $V_{1}$ and $V_{2}$ are symplectic spaces with respect to forms $f_{1}$ and $f_{2}$ respectively, then a linear map $\alpha: V_{1} \rightarrow V_{1}$ is called an isometry if it is non-singular and satisfies $f_{1}(u, v)=f_{2}(\alpha(u), \alpha(v))$.

We shall show that a non degenerate symplectic space $V$ over $K$ is uniquely determined up to isometries by $\operatorname{dim}_{K}(V)$. In order to do this we need the following definition.

Definition 3.4. Let $V$ be a symplectic space over $K$ with respect to the form $f$. An hyperbolic plane of $V$ is a 2-dimensional subspace $H=<v_{1}, v_{2}>$ such that $f\left(v_{1}, v_{2}\right)=1$.

We can now prove the following.

Proposition 3.1.3. Let $V$ be a non-degenerate symplectic space over $K$ of dimension $n$ with respect to the form $f$. Then $V$ is unique up to isometries. 
Proof. If $V$ is non-degenerate, then $V$ contains an hyperbolic plane. In fact, for every $w \neq 0$ of $V$, there exists an element $v \in V$ such that $f(w, u) \neq 0$. In particular, choosing $w_{1} \in V \backslash\{0\}$, there exists $v_{1} \in V$ such that $f\left(w_{1}, v_{1}\right)=1$ and $H_{1}:=<w_{1}, v_{1}>$ is an hyperbolic plane.

Now we consider the space $V^{\prime}:=H_{1}^{\perp}$. We want to prove that $V^{\prime}$ is a nondegenerate symplectic space with respect to the form $f^{\prime}=\left.f\right|_{V^{\prime}}$. We have that $V^{\prime}$ is symplectic, being a subspace of the symplectic space $V$. Moreover it is easy to see that

$$
V^{\prime \perp}=\left\{w \in V^{\prime} \mid f^{\prime}(w, u)=0 \forall u \in V^{\prime}\right\}
$$

is contained in $V^{\perp}=0$. Therefore $V^{\prime}$ contains an hyperbolic plane $H_{2}$. Proceeding in this way, we get that $V$ can be written as a direct sum of its hyperbolic planes $H_{1}, \ldots H_{t}$ with $H_{i} \perp H_{j}$ for $1 \leq i \neq j \leq t$. Thus $V$ has even dimension $2 t$.

Since an isometry between symplectic spaces brings hyperbolic planes into hyperbolic planes, from the decomposition

$$
V=H_{1} \oplus H_{2} \oplus \ldots \oplus H_{t}
$$

it follows that $V$ is determined up to isometries by its dimension $n$ over $K$.

The next definition will be useful to study extraspecial groups of even order.

Definition 3.5. Let $K$ be a field of characteristic 2, $V$ a finite dimensional vector space over $K$ and $f: V \times V \rightarrow K$ a bilinear form on $V$. A quadratic form on $V$ (associated to $f$ ) is a map $q: V \rightarrow K$ which satisfies

$$
q(a u+b v)=a^{2} q(u)+b^{2} q(v)+a b f(u, v)
$$

for all $a, b \in K$ and $u, v \in V$.

Remark 3.1. Let $V$ be a finite dimensional vector space over $K$ with $\operatorname{char}(K)=$ 2 with a bilinear form $f$ and a quadratic form $q$ associated to $f$. Then $V$ is a symplectic space with respect to $f$.

Proof. We have to show that $f(v, v)=0$ for every $v \in V$. We consider equation 3.1 with $a=b=1$ and $u=v$. Then $f(v, v)=q(2 v)-2 q(v)=0$ for all $v \in V$ since $\operatorname{char}(K)=2$.

Definition 3.6. A quadratic form $q$ on $V$ is called non degenerate if $V$ is a non degenerate symplectic space.

\subsubsection{Connection between symplectic spaces and extraspe- cial $p$-groups}

We shall now describe the relation between vector spaces endowed with forms and extraspecial $p$-groups through the following lemma.

Lemma 3.1.4. Let $E$ be an extraspecial p-group, with $Z(E)=<z>$, and consider $V:=E / Z(E)$ as a vector space over $\mathbb{F}_{p}$. 
(1) Define a map $f: V \times V \rightarrow \mathbb{F}_{p}$ such that $f(\bar{x}, \bar{y})=a$ if $[x, y]=z^{a}$, where $x$ and $y$ are representatives in $E$ of $\bar{x}$ and $\bar{y}$ respectively. Then $f$ is a welldefined bilinear form with respect to which $V$ is a non-degenerate symplectic space.

(2) If $p=2$ the map $q: V \rightarrow \mathbb{F}_{2}$ given by $q(\bar{x})=b$ if $\bar{x}=x Z(E)$ and $x^{2}=z^{b}$ is a non-degenerate quadratic form on $V$ associated with $f$.

Proof. (1) We first notice that $f$ is well defined since the commutator $[x, y]$ depends only on the cosets and not on the coset representatives. Moreover $f$ is bilinear. In fact, since $E^{\prime}=Z(E)$, given $x, w, y \in E$ we have that

$$
[x w, y]=x w y w^{-1} y^{-1} x^{-1}[x, y]=x[w, y] x^{-1}[x, y]=[x, y][w, y]
$$

and similarly $[x, w y]=[x, w][x, y]$.

Now we want to prove that $V$ is symplectic and non-degenerate. We have that $f(\bar{x}, \bar{x})=0$ for all $\bar{x} \in V$ since $[x, x]=1=z^{0}$ for all $x \in E$; moreover if $\bar{x} \in V^{\perp}$, with $\bar{x}=x Z(E)$, then $[x, y]=1$ for all $y \in E$, hence $x \in Z(E)$ and $V^{\perp}=\{0\}$.

(2) Let $p=2$. We notice that the definition of the map $q$ makes sense since, if $x, y \in E$, then $[x, y] \in Z(E)$ and $[x, y]^{2}=1$ and we have

$$
x^{2} y=x(x y)=x[x, y] y x=[x, y](x y) x=[x, y]^{2}(y x) x=y x^{2},
$$

hence $x^{2} \in Z(E)$ for all $x \in E$.

The map $q$ is well defined, since $q(\bar{x})$ depends only on $\bar{x}$. Moreover $q$ is a non-degenerate quadratic form associated with $f$. In fact given $a, c \in \mathbb{F}_{2}$ and $\bar{x}, \bar{y} \in E / Z(E)$. Then $q(a \bar{x}+c \bar{y})=b$ if and only if $z^{b}=\left(x^{a} y^{c}\right)^{2}$ and, since $\left(x^{a}\right)^{2}$ and $\left(y^{c}\right)^{2}$ are central elements, we have

$$
z^{b}=\left(x^{a} y^{c}\right)^{2}=x^{a} y^{c}\left(x^{a}\right)^{2} x^{-a}\left(y^{c}\right)^{2} y^{-c}=\left(x^{a}\right)^{2}\left(y^{c}\right)^{2}\left[x^{a}, y^{c}\right] .
$$

Now $\left[x^{a}, y^{c}\right]=[x, y]^{a c}$ and $a^{2}=a$ and $c^{2}=c$ for every $a, c \in \mathbb{F}_{2}$, thus we can write

$$
z^{b}=\left(x^{2}\right)^{a}\left(y^{2}\right)^{c}[x, y]^{a c}=z^{a^{2} q(\bar{x})+c^{2} q(\bar{y})+a c f(\bar{x}, \bar{y})}
$$

getting $q(a \bar{x}+c \bar{y})=a^{2} q(\bar{x})+c^{2} q(\bar{y})+a c f(\bar{x}, \bar{y})$.

We end this section with a result which is a small step towards the proof of property (i) and the classification of extraspecial groups. In order to state it, we recall the definition of central product of groups.

Definition 3.7. A group $G$ is said to be a central product of its subgroups $U_{1}, \ldots, U_{n}$ if $G=U_{1} \ldots U_{n}$ as a set and $\left[U_{i}, U_{j}\right]=1$ for $i \neq j$.

We have the following result. 
Proposition 3.1.5. Let $E$ be an extraspecial p-group. Then $E$ has order $p^{2 m+1}$ for some positive integer $m$ and $E$ is a central product of $m$ extraspecial groups of order $p^{3}$.

Proof. In view of Lemma 3.1.4, $E / Z(E)$ is a non-degenerate symplectic space endowed with a form $f$ and from Proposition 3.1.3 it can be written as a direct sum of hyperbolic planes

$$
E / Z(E)=H_{1} \oplus H_{2} \oplus \ldots \oplus H_{m}
$$

and $H_{i} \perp H_{j}$ for $1 \leq i \neq j \leq t$. We denote by $E_{i}$ the inverse image of $H_{i}$ under the projection map $\pi: E \rightarrow E / Z(E)$. Then $E_{i}$ is generated by two elements $x, y \in E$ such that $H_{i}=<\pi(x), \pi(y)>$ and, since $H_{i}$ is an hyperbolic plane, we have that $f(\pi(x), \pi(y))=1$. Thus the commutator $[x, y]=z$ generates the center $Z(E)$.

We want to prove that $E_{i}$ is an extraspecial $p$-group. We notice that $E_{i}$ has order $p^{3}$ since $\left|E_{i}\right|=\left|H_{i}\right||Z(E)|=p^{3}$ and it is non abelian since it has non trivial commutator subgroup. Moreover $1 \neq E_{i}^{\prime} \leq E^{\prime}=Z(E)$, thus $E_{i}^{\prime}=Z(E)$ has order $p$. Now if $Z\left(E_{i}\right)$ had order $p^{2}$, then the group $E_{i} / Z\left(E_{i}\right)$ would be cyclic and thus $E_{i}$ would be abelian, which is a contradiction. Then $E_{i} / Z\left(E_{i}\right)$ is elementary abelian, having order $p^{2}$, which implies that $\Phi\left(E_{i}\right)=E_{i}^{\prime}=Z\left(E_{i}\right)$ are groups of order $p$ and $E_{i}$ is extraspecial.

Since $H_{i} \perp H_{j}$ for $i \neq j$, in terms of the $E_{i}$ 's we have $\left[E_{i}, E_{j}\right]=1$ for $i \neq j$. Hence we have proved that $E$ is a central product of its subgroups $E_{1}, \ldots, E_{m}$ which are extraspecial $p$-groups of order $p^{3}$.

Remark 3.2. It is not true in general that a central product of extraspecial groups is extraspecial. We take, as an example, the direct product of $m$ extraspecial groups. It is a central product of them, but the center is the direct product of the centers and it has order $p^{m}$.

However, we have the following condition.

Proposition 3.1.6. Let $G$ be a central product of extraspecial p-groups $E_{1}, \ldots, E_{m}$. Then $G$ is extraspecial if and only if $Z(G)=\bigcap_{i=1}^{m} Z\left(E_{i}\right)$.

Proof. Suppose that $G$ is extraspecial. Since $\left[E_{i}, E_{j}\right]=1$ for all $i \neq j$, we have that $Z\left(E_{i}\right)=Z\left(E_{j}\right)$ for every $i, j$ and moreover $Z\left(E_{i}\right)=Z(G)$ since they have the same order.

Conversely suppose that $Z(G)=\bigcap_{i=1}^{m} Z\left(E_{i}\right)$. Then $Z\left(E_{i}\right) \subseteq Z(G)=$ $\bigcap_{j=1}^{m} Z\left(E_{j}\right) \subseteq Z\left(E_{i}\right)$ and thus $Z(G)=Z\left(E_{i}\right)$ for all $i$ 's and it is cyclic of order $p$. Now if $g \in G^{\prime}$, then $g=\left[v_{1} \cdot \ldots \cdot v_{m}, w_{1} \cdot \ldots \cdot w_{m}\right]$ with $v_{i}, w_{i} \in E_{i}$ for every $i \in\{1, \ldots, m\}$, and since $\left[E_{i}, E_{j}\right]=1$ for $i \neq j$ we have

$$
g=\left[v_{1}, w_{1}\right] \cdot \ldots \cdot\left[v_{m}, w_{m}\right] .
$$

Then $G^{\prime}$ is a central product of the groups $E_{i}^{\prime}$ 's and since $E_{i}^{\prime}=Z\left(E_{i}\right)$ we get $G^{\prime}=Z(G)$ and they have both order $p$.

We shall see that, in this case, the central product is said to be amalgamated on the centers and we largely discuss it in section 3.2.1. 


\subsection{Classification of extraspecial $p$-groups}

This section is devoted to study the structure of extraspecial groups in relation to their orders and exponents. We shall see how these groups can be explicitly constructed starting from extraspecial groups of order $p^{3}$. In order to do this we have to define a special central product amalgamated on the centers.

\subsubsection{Central products of groups amalgamated on the cen- ters}

We have seen in Proposition 3.1.5 that every extraspecial group is a central product of groups of order $p^{3}$ and in Proposition 3.1.6 a condition for a central product of extraspecial groups to be extraspecial was given. However these results are not effective, in the sense that given a family of groups of order $p^{3}$ it is not clear which extraspecial group could be built up from it.

Now, we want to make a construction which is, in some sense, the dual of Definition 3.7, that is, given a finite number of extraspecial groups, we want to explicitly construct a central product of them in such a way that this product is also an extraspecial group.

We suppose to have $n$ groups $G_{1}, \ldots, G_{n}$ such that $Z\left(G_{i}\right) \simeq Z\left(G_{j}\right)$ for all $i, j$; we suppose moreover to fix, for every $i$, an isomorphism $\mu_{i}: A \rightarrow Z\left(G_{i}\right)$ where $A$ is an abelian group. We set $D:=G_{1} \times \ldots \times G_{n}$ and let

$$
\bar{G}_{i}=\left\{\left(1, \ldots, 1, g_{i}, 1, \ldots, 1\right) \mid v_{i} \in G_{i}\right\} \simeq G_{i}
$$

denote the $i$-th coordinate subgroup of $D$.

We consider the subgroup $N$ of $D$ defined as

$$
N:=\left\{\left(\mu_{1}\left(a_{1}\right), \ldots, \mu_{n}\left(a_{n}\right)\right) \mid a_{i} \in A, \prod_{i=1}^{n} a_{i}=1\right\} .
$$

We have the following proposition.

Proposition 3.2.1. The group $N$ is normal in $D$ and $\bar{G}_{i} \cap N=1$.

Proof. We have that $N \subseteq Z(D)$ thus it is normal. Moreover

$$
\bar{G}_{i} \cap N=\left\{\left(1, \ldots, 1, g_{i}, 1, \ldots, 1\right) \mid v_{i}=\mu_{i}\left(a_{i}\right), a_{i}=1\right\}=1 .
$$

Now we want to prove that the quotient $D / N$ is isomorphic to a central product of the $G_{i}$ 's.

Proposition 3.2.2. Let $G:=D / N$ and $U_{i}:=\bar{G}_{i} N / N \leq G$. Then $G$ is a central product of the groups $U_{i}$ 's and $U_{i} \simeq G_{i}$ for all $i$ 's.

Moreover, setting $A_{i}:=\left\{\left(1, \ldots, \mu_{i}(a), \ldots, 1\right) \mid a \in A\right\} \leq \bar{G}_{i}$, we have

$$
U_{i} \cap U_{j}=\bigcap_{k=1}^{n} U_{k}=A_{i} N / N \simeq A
$$


for every $1 \leq i \neq j \leq n$.

Proof. First of all we have to prove that $G=U_{1} \ldots U_{n}$ and $\left[U_{i}, U_{j}\right]=1$ if $i \neq j$. Since $N<Z(D)$ and $\bar{G}_{i}$ and $\bar{G}_{j}$ commutes, then $\left[U_{i}, U_{j}\right]=1$. Now we notice that every element $g=\left[\left(g_{1}, \ldots, g_{n}\right)\right]_{N} \in G$ with $g_{i} \in G_{i}$ can be written as a product of elements of the $U_{i}$ 's, that is $g=u_{1} \ldots u_{n}$ where

$$
u_{i}:=\left[\left(1, \ldots, g_{i}, \ldots, 1\right)\right]_{N}=\left[\left(\mu_{1}(1), \ldots, g_{i} \mu_{i}(1), \ldots, \mu_{n}(1)\right)\right]_{N} \in U_{i} .
$$

It is easy to see that the map $\phi_{i}: U_{i} \rightarrow G_{i}$ defined as

$$
\phi_{i}\left[\left(\mu_{1}\left(a_{1}\right), \ldots, v_{i} \mu_{i}\left(a_{i}\right), \ldots, \mu_{n}\left(a_{n}\right)\right)\right]_{N}=v_{i}
$$

is an isomorphism; so we are left to prove the condition on the intersection of the $U_{i}$ 's. Let $u \in U_{i} \cap U_{j}, i \neq j$. Then

$$
u=\left[\left(\left(\mu_{1}\left(a_{1}\right), \ldots, v_{i} \mu_{i}\left(a_{i}\right), \ldots, \mu_{n}\left(a_{n}\right)\right)\right)\right]_{N}
$$

with $\prod_{i} a_{i}=1$ and $v_{i} \in Z\left(G_{i}\right)$, that is $v_{i}=\mu_{i}(a)$ for some $a \in A$. We notice that we can write $u$ as

$u=\left[\left(1, \ldots, \mu_{k}(a), \ldots, 1\right)\left(\mu_{1}\left(a_{1}\right), \ldots, \mu_{i}\left(a a_{i}\right), \ldots, \mu_{k}\left(a^{-1} a_{k}\right), \ldots, \mu_{n}\left(a_{n}\right)\right)\right]_{N} \in U_{k}$

thus $u \in \bigcap_{k} U_{k}=U_{i} \cap U_{j}$. Moreover $U_{i} \cap U_{j} \subseteq A_{i} N / N \leq \bar{G}_{i}$ and it easy to see that the inverse inclusion holds. Finally the map $\varphi_{i}: A_{i} N / N \rightarrow A$ defined by

$$
\varphi_{i}\left[\left(\mu_{1}\left(a_{1}\right), \ldots, \mu_{i}(a) \mu_{i}\left(a_{i}\right), \ldots, \mu_{n}\left(a_{n}\right)\right)\right]_{N}=a
$$

is clearly an isomorphism.

We can now give the following definition.

Definition 3.8. The constructed group $G=D / N$ is said to be a central product of $G_{1}, \ldots, G_{n}$ amalgamated on the centers, or simply amalgamated central product, and we denote it by $G=G_{1} \curlyvee \ldots \curlyvee G_{n}$.

Remark 3.3. We notice that the isomorphism type of $G$ is not uniquely determined since it depends on the choice of the $\mu_{i}$ 's. Thus the notation $G_{1} \curlyvee \ldots \curlyvee G_{n}$ stands for any one class of such central products.

The next remark clarifies when a central product of groups with isomorphic centers is amalgamated on the centers.

Proposition 3.2.3. Let $G$ be a central product of $G_{1}, \ldots, G_{n}$ and suppose that $Z\left(G_{i}\right) \simeq Z\left(G_{j}\right)$ for all $i \leq j$. Then $G$ is isomorphic to an amalgamated central product if and only if $Z(G)=\bigcap_{i=1}^{n} Z\left(G_{i}\right)$.

Proof. Suppose that $G$ is isomorphic to an amalgamated central product of the $G_{i}^{\prime}$ s, say $\simeq G_{1} \curlyvee \ldots \curlyvee G_{n}$, via the isomorphism

$$
\mu: H=G_{1} \curlyvee \ldots \curlyvee G_{n} \rightarrow G=G_{1} \ldots G_{n} .
$$


By construction $H$ is a central product of $U_{1}, \ldots, U_{n}$ with $U_{i}=\bar{G}_{i} N / N \simeq G_{i}$ and $\mu\left(U_{i}\right)=G_{i}$. Now $Z\left(U_{i}\right)=Z\left(\bar{G}_{i}\right) N / N$. In fact $u_{i} \in Z\left(U_{i}\right)$ if and only if

$$
u_{i}=\left[\left(\mu_{1}\left(a_{1}\right), \ldots, v_{i} \mu_{i}\left(a_{i}\right), \ldots, \mu_{n}\left(a_{n}\right)\right)\right]_{N}
$$

with $\prod_{i=1}^{n} a_{i}=1$ and $v_{i} \in Z\left(G_{i}\right)$.

Since $H$ is a central product of the $U_{i}$ 's, we have that $Z\left(U_{i}\right) \subseteq Z(H)$. Now, if $g \in Z(H)$, then $g=u_{1} \ldots u_{n}$ with $u_{i} \in Z\left(U_{i}\right)$, hence

$$
g=\left[\left(z_{1} \mu_{1}\left(a_{1}\right), \ldots, z_{i} \mu_{i}\left(a_{i}\right), \ldots, z_{n} \mu_{n}\left(a_{n}\right)\right)\right]_{N}
$$

with $\prod_{i=1}^{n} a_{i}=1$ and $z_{i} \in Z\left(G_{i}\right)$. Thus there exist elements $b_{i} \in A$ such that $z_{i}=\mu_{i}\left(b_{i}\right)$ for every $i$ and, setting $c=\left(b_{1} \ldots b_{i-1} b_{i+1} \ldots b_{n}\right)^{-1}$, we get

$$
g=\left[\left(1, \ldots, z_{i} \mu_{i}(c), \ldots, 1\right)\left(\mu_{1}\left(b_{1} a_{1}\right), \ldots, \mu_{i}\left(c^{-1} a_{i}\right), \ldots, \mu_{n}\left(b_{n} a_{n}\right)\right)\right]_{N} .
$$

Therefore $g \in Z\left(U_{i}\right)$ and $Z(H)=\bigcap_{i=1}^{n} Z\left(U_{i}\right)$. Finally we get that $Z(G)=$ $\mu(Z(H))=\bigcap_{i=1}^{n} Z\left(G_{i}\right)$.

Conversely, suppose that $G$ is a central product of $G_{1}, \ldots, G_{n}$, with $Z\left(G_{i}\right) \simeq$ $Z\left(G_{j}\right)$ and $Z(G)=\bigcap_{i=1}^{n} Z\left(G_{i}\right)$. Then $Z(G) \simeq Z\left(G_{i}\right)$ for every $i$, since $Z\left(G_{i}\right) \subseteq$ $Z(G) \subseteq Z\left(G_{i}\right)$.

We fix isomorphisms $\mu_{i}: A=Z(G) \rightarrow Z\left(G_{i}\right)$ and we consider the amalgamated product $H=G_{1} \curlyvee \ldots \curlyvee G_{n}$ constructed with this choice of the $\mu_{i}$ 's.

Now the map $\mu: G \rightarrow H$ defined by

$$
\mu\left(v_{1} \ldots v_{n}\right)=\left[\left(v_{1}, \ldots, v_{n}\right)\right]_{N}
$$

is clearly a well defined and surjective homomorphism, with kernel

$$
\operatorname{Ker} \mu=\left\{\left(v_{1} \ldots v_{n}\right) \mid v_{i}=\mu_{i}\left(a_{i}\right), \prod_{i} a_{i}=1\right\} \in Z(G)=\bigcap_{i=1}^{n} Z\left(G_{i}\right) .
$$

Hence, if $v \in \operatorname{Ker} \mu$, then $v \in Z\left(G_{i}\right)$ for every $i$, thus $v=\mu_{i}\left(a_{i}\right)$ with $a_{i}=1$ and $v=1$. Therefore $\mu$ in also injective and it is an isomorphism. lary.

Proposition 3.2.3 together with Proposition 3.1.6 give us the following corol-

Corollary 3.2.4. Let $G$ be a central product of extraspecial p-groups. Then $G$ is extraspecial if and only if $G$ is isomorphic to a central product of those groups amalgamated on the centers.

\subsubsection{Extra-special $p$-groups of order $p^{3}$}

We have seen that every extraspecial $p$-group can be constructed as an amalgamated central product of a finite number of extraspecial groups of order $p^{3}$ and conversely every amalgamated central product of extraspecial groups of order $p^{3}$ is extraspecial. We are now concerned with the description and the classification of extraspecial $p$-groups of order $p^{3}$. We start with the following easy remark. 
Remark 3.4. Every non-abelian group of order $p^{3}$ is extraspecial.

Proof. Let $G$ be a non-abelian group with $|G|=p^{3}$. Then $|Z(G)|=p$. In fact $|Z(G)| \notin\left\{1, p^{3}\right\}$ since the center of a $p$-group is nontrivial and $G$ is not abelian. If we suppose $|Z(G)|=p^{2}$, we get that $|G / Z(G)|=p, G / Z(G)$ is cyclic and therefore $G$ would be abelian, which is a contradiction.

Now $G / Z(G)$ is abelian, since it has order $p^{2}$ (every group of order $p^{2}$ is abelian). Hence we have $\left|G^{\prime}\right|=p$ since $G^{\prime}$ is the smallest normal subgroup of $G$ such that $G / G^{\prime}$ is abelian.

The following theorem states that, for every prime $p$, there are only two types of extraspecial groups of order $p^{3}$, up to isomorphisms.

Theorem 3.2.5. Let $E$ be an extraspecial p-group of order $p^{3}$.

If $p=2$ then $E$ is isomorphic to either the dihedral group $D$ or the quaternion group $Q$, where $D$ and $Q$ have the following presentation

$$
\begin{gathered}
D=<a, b \mid a^{2}=b^{2}=(a b)^{4}=1>, \\
Q=<a, b \mid a^{2}=b^{2}, b^{-1} a b=a^{-1}, a^{4}=1>.
\end{gathered}
$$

If $p$ is odd, then $E$ is isomorphic to either the group $H$ or the group $F$ where $H$ and $F$ are the groups with the following presentation

$$
\begin{gathered}
H=<x, y \mid x^{p}=y^{p}=1,[x, y] \in Z(H)>, \\
F=<x, y \mid x^{p^{2}}=y^{p}=1,[x, y]=x^{p}>.
\end{gathered}
$$

Proof. Let $p=2$. In this case we know that $Z(E)=<z>$ with $z^{2}=1, E$ is generated by two elements $a$ and $b$ with $[a, b]=z$ and, from Lemma 3.1.1, $x^{2} \in Z(E)=\{1, z\}$ for every $x \in E$. Moreover we notice that $E=<a, b>=<$ $a, a b>$. We now distinguish three cases:

1. if $a^{2}=b^{2}=1$, then $(a b)^{4}=(a b a b)(a b a b)=z^{2}=1$ and $E \simeq D$;

2. if $a^{2}=z$ and $b^{2}=1$, then, since $a^{2}=[a, b]$, we have $(a b)^{2}=a^{2} b^{2} a^{2} b=$ $z^{2}=1$ and again we get $E=<a b, b>\simeq D$;

3. if $a^{2}=b^{2}=z$, we have $a^{-1}=b^{-1} a b$ and $a^{4}=1$, thus $E \simeq Q$.

Let $p$ be an odd prime. In this case $Z(E)=\langle z\rangle$ with $z^{p}=1$ and $E=\langle x, y\rangle$ with $[x, y]=z$. Moreover, again from Lemma 3.1.1, we have that $x^{p}, y^{p} \in Z(E)$ since $\left[x^{p}, y\right]=[x, y]^{p}=\left[x, y^{p}\right]=1$. Here different cases occur:

1. if $x^{p}=y^{p}=1$ then, since by hypothesis $[x, y]$ generates $Z(E)$, we have $E \simeq H$

2. if $x^{p} \neq 1$, then $x^{p}$ is a generator for $Z(E)$ and $x^{p}=[x, y]^{a}=\left[x, y^{a}\right]$ for some $1 \leq a<p$. Now, since $(a, p)=1$, there exist $r, s \in \mathbb{Z}$ such that $r a+s p=1$ and $y^{p}=\left(x^{p}\right)^{m}$ for some integer $m$. So $y=y^{r a+p s}=$ $\left(y^{a}\right)^{r}\left(x^{p}\right)^{m s} \in<x, y^{a}>$ thus $E=<x, y^{a}>$. Hence we can choose the generators $x$ and $y$ of $E$ in such a way that $x^{p}=[x, y]$. We now have two cases: 
(a) if $y^{p}=1$, then $E \simeq F$;

(b) if $y^{p} \neq 1$, then $y^{p}$ also generates $Z(E)$ and there exists $n \in \mathbb{Z}$ such that $y^{p}=\left(x^{p}\right)^{n}$. If we set $w=\left(y x^{-n}\right)$, then $w^{p}=1$. In fact, using Proposition 3.1.2, we get $w^{p}=\left(y x^{-n}\right)^{p}=y^{p} x^{-n p}=1$. Then $E=<x, w>$ and, as before, there exists $1 \leq l<p$ such that $E=<x, w^{l}>$ and $x^{p}=\left[x, w^{l}\right]$ with $\left(w^{l}\right)^{p}=1$. Thus we get $E \simeq F$.

We end this subsection with another description of extraspecial groups of order $p^{3}$, which shall be useful to understand how to realize them as Galois groups of some extension of number fields. We recall the following definition:

Definition 3.9. Given two groups $G$ and $H$ and a group morphism $\varphi: H \rightarrow$ Aut $(G)$, the semidirect product of $G$ and $H$ via $\varphi$ is a group $X$, denoted by $G \rtimes_{\varphi} H$, such that:

(i) $X=G \times H$ as a set;

(ii) the group law is given by $(g, h) \circ_{X}\left(g^{\prime}, h^{\prime}\right):=\left(g \circ_{G} \varphi\left(h^{-1}\right)\left(g^{\prime}\right), h \circ_{H} h^{\prime}\right)$, where ${ }^{\circ} G$ and ${ }^{\circ} H$ are the group operations in $G$ and $H$, respectively.

Remark 3.5. It is easy to show that $X=G \rtimes_{\varphi} H$ is a group, with identity $1_{X}=$ $\left(1_{G}, 1_{H}\right)$ and the inverse of the element $(g, h)$ is $\left(\varphi\left(h^{-1}\right)\left(g^{-1}\right), h^{-1}\right)$. Obviously the subgroup $G \rtimes\left\{1_{H}\right\} \simeq G$ is normal in $X$.

We now want to prove that the groups $H$ and $F$ of Theorem 3.2.5 can be viewed as semidirect products of two abelian groups, as the following proposition states.

Proposition 3.2.6. Let $p$ be an odd prime number and let $H$ and $F$ denote the two non abelian groups of order $p^{3}$. Consider the groups

$$
A:=(\mathbb{Z} / p \mathbb{Z})^{2} \rtimes_{\varphi} \mathbb{Z} / p \mathbb{Z}
$$

and

$$
B:=\left(\mathbb{Z} / p^{2} \mathbb{Z}\right) \rtimes_{\vartheta} \mathbb{Z} / p \mathbb{Z}
$$

where

$$
\begin{gathered}
\varphi: \mathbb{Z} / p \mathbb{Z} \rightarrow \operatorname{Aut}\left((\mathbb{Z} / p \mathbb{Z})^{2}\right)=G L(2, p) \\
\varphi\left([c]_{p}\right)=\left(\begin{array}{ll}
1 & 0 \\
c & 1
\end{array}\right)
\end{gathered}
$$

and

$$
\begin{gathered}
\vartheta: \mathbb{Z} / p \mathbb{Z} \rightarrow \operatorname{Aut}\left(\mathbb{Z} / p^{2} \mathbb{Z}\right) \\
\vartheta\left([c]_{p}\right)\left(\left([a]_{p^{2}}\right)=\left([(p c+1) a]_{p^{2}}\right) .\right.
\end{gathered}
$$

Then $A \simeq H$ and $B \simeq F$.

Moreover, if $p=2$ and $D$ is the dihedral group of order 8 , then $A \simeq B \simeq D$. 
Proof. It is easy to check that $\varphi$ is a well-defined group morphism. Now we consider two elements of $A, \alpha=((1,0), 0)$ and $\beta=((0,0), 1)$. We notice that $\alpha^{m}=((m, 0), 0)$ and $\beta^{m}=((0,0), m)$ for every $m \in\{1, \ldots, p\}$. Hence $\alpha$ and $\beta$ have order $p$ and $\alpha \beta=((1,0), 1) \neq((1,-1), 1)=\beta \alpha$. Thus $A$ is a non-abelian group of order $p^{3}$. Moreover we have that if $((a, b), c) \in A$, then $((a, b), c)^{m}=((m a, m b-m(m-1) a c / 2), m c)$ hence $\exp (A)=p$. From Theorem 3.2 .5 we have that $A \simeq H$. We notice that $Z(A)=<z>$ where $z=((0,1), 0)$ and it has order $p$. Moreover $[\alpha, \beta]=((0,1), 0)$. So an explicit isomorphism is given by $\phi: H \rightarrow A$ with $\phi(x)=\alpha$ and $\phi(y)=\beta$.

As before, we observe that $\vartheta$ is a well-defined action of $\mathbb{Z} / p \mathbb{Z}$ on $\mathbb{Z} / p^{2} \mathbb{Z}$. Now let $\gamma=(1,0), \delta=(0,1) \in B$. We have that $\gamma \delta=(1,1) \neq \delta \gamma=(1-p, 1)$. Hence $B$ is non-abelian and $|B|=p^{3}$. Moreover we have that $(a, b)^{m}=(a(2 m-$ $m(m-1) p b) / 2, m b)$ for every $(a, b) \in B$ and $m \in \mathbb{Z}$. Thus $\exp (B)=p^{2}$, since $\delta^{p}=(0,0)$, but $\gamma^{p}=(p, 0) \neq(0,0)$, and, by Theorem 3.2.5, we have that $B \simeq F$. An explicit isomorphism is $\phi: F \rightarrow B$ with $\phi(x)=\gamma, \phi(y)=\delta$ (we also notice that $\left.\gamma^{p}=[\gamma, \delta]\right)$.

If $p=2$, then the map

$$
\lambda:(\mathbb{Z} / 2 \mathbb{Z})^{2} \rtimes \mathbb{Z} / 2 \mathbb{Z} \rightarrow(\mathbb{Z} / 4 \mathbb{Z}) \rtimes \mathbb{Z} / 2 \mathbb{Z}
$$

with $\lambda\left(\left([a]_{2},[b]_{2}\right),[c]_{2}\right)=\left([a+2 b]_{4},[c]_{2}\right)$ is an isomorphism between $A$ and $B$. These groups are non abelian groups of order 8 and they cannot be isomorphic to the quaternion group $Q$, since $Q$ cannot be written as a semidirect product of two groups. In fact every proper subgroup of $Q$ is normal and abelian (it has order 2 or 4) and if $Q$ was a semidirect product of its normal subgroups, then it would be a direct product of them and so $Q$ would be abelian. Then they are both isomorphic to the dihedral group $D$ of order 8 .

\subsubsection{The structure theorem for extraspecial $p$-groups}

In order to prove the structure theorem for extraspecial $p$-groups, we need the following lemma which describes when two different amalgamated central products of extraspecial groups of order $p^{3}$ are isomorphic.

Lemma 3.2.7. Let $p$ be an odd prime and let $H$ and $F$ be the extraspecial groups of order $p^{3}$ defined in Theorem 3.2.5. Then $F \curlyvee F \simeq H \curlyvee F$. Moreover, if $p=2$ and $D$ and $Q$ are as in Theorem 3.2.5, then $D \curlyvee D \simeq Q \curlyvee Q$.

Proof. Let $G \simeq F \curlyvee F$. From Proposition 3.2.2, we can write $G=F_{1} F_{2}$ with $\left[F_{1}, F_{2}\right]=1$ and $F_{i}=\left\langle x_{i}, y_{i}\right\rangle$ with $x_{i}, y_{i}$ satisfying the relations for the generators of $F$ given in Theorem 3.2.5, $\forall i \in\{1,2\}$.

Since $\left.Z\left(F_{i}\right)=<\left[x_{i}, y_{i}\right]\right\rangle=\left\langle x_{i}^{p}\right\rangle$, replacing $x_{2}$ by some suitable power, we can suppose that $\left(x_{1} x_{2}\right)^{p}=1$. In fact, from Corollary 3.2.4, $G$ is extraspecial and $Z(G)$ is cyclic of order $p$. Since $x_{2}^{p}, x_{1}^{p} \in Z(G)$, we have that they both generate $Z(G)$, hence there exists $m \in \mathbb{Z}$ such that $x_{2}^{p m}=x_{1}^{p}$ with $0<m<p$ and $\left(x_{1}, x_{2}^{-m}\right)^{p}=1$. Now we consider the group $U=<x_{1} x_{2}, y_{1}>$ and we claim 
that $U$ is a subgroup of $G$ isomorphic to $H$. In fact $U$ is generated by two elements of order $p$ and, since $\left[F_{1}, F_{2}\right]=1$, we have that their commutator

$$
u=\left[x_{1} x_{2}, y_{1}\right]=x_{1} x_{2} y_{1} x_{2}^{-1} x_{1}^{-1} y_{1}^{-1}=\left[x_{1}, y_{1}\right]
$$

generates the center $Z\left(F_{1}\right)$, therefore $u y_{1}=y_{1} u$ and, since $\left[F_{1}, F_{2}\right]=1, u x_{1} x_{2}=$ $x_{1} u x_{2}=x_{1} x_{2} u$. So $u \in Z(U)$ and $U \simeq H$.

Now we consider the quotient $V=G / Z(G)$. Since $Z(G)=Z\left(F_{1}\right) \cap Z\left(F_{2}\right)$, then $Z(G) \subseteq Z(U)$. The subspace $U / Z(G)$ is an hyperbolic plane of $V$ : in fact $U$ is generated by two elements whose commutator generates $Z(U)$ and $U / Z(G)$ is generated by their images in the quotient. Hence there exists a subgroup $L$ of $G$ such that $V=(U / Z(G)) \perp(L / Z(G))$, where $L / Z(G)$ is the hyperbolic plane orthogonal to $U / Z(G)$.

We claim that $L$ is an extraspecial group of order $p^{3}$ isomorphic to $F$. In fact $\exp (L)=p^{2}$, otherwise we would have $\exp (G)=p$, which is a contradiction since $G$ contains elements of order $p^{2}$ (e.g. $x_{1}$ ). Thus $L \simeq F$ and $G$ is isomorphic to a central product of $H$ and $F$. From Corollary 3.2.4, since $G$ is extraspecial, we have $G \simeq H \curlyvee F$.

In a similar way it can be proved that $Q \curlyvee Q \simeq D \curlyvee D$.

We can now state the structure theorem for extraspecial $p$-groups.

Theorem 3.2.8. Let $p$ be a prime number and let $H, F, D$ and $Q$ be the groups defined in Theorem 3.2.5. Let $E$ be an extraspecial $p$-group of order $p^{2 m+1}$ for some positive integer $m$. Then $E$ has the following structure:

1. if $p \neq 2$ and $\exp (E)=p, E$ is a central product of $m$ copies of $H$;

2. if $p \neq 2$ and $\exp (E)=p^{2}, E$ is a central product of $m-1$ copies of $H$ and one copy of $F$;

3. if $p=2$ then:

(a) either $E$ is a central product of $m$ copies of $D$;

(b) either $E$ is a central product of $m-1$ copies of $D$ and one copy of $Q$.

Proof. We already proved that in any case $E=E_{1} \ldots E_{m}$ is a central product of $m$ extraspecial subgroups $E_{i}$ of order $p^{3}$ and so, by Proposition 3.2.4,E $\simeq$ $E_{1} \curlyvee \ldots \curlyvee E_{m}$.

1. If $p$ is odd and $\exp (E)=p$, then $E_{i} \simeq H$ for all $i$.

2. If $p$ is odd and $\exp (E)=p^{2}$, then we have $E_{i} \simeq F$ for at least one index $i$. We can suppose that $E$ is a central product of $t$ copies of $F$ and $m-t$ copies of $H$, with $m \geq 1$. Then, by Lemma 3.2.7, we can prove, by induction on $t$, that a central product of $t$ copies of $F$ is isomorphic to a central product of $t-1$ copies of $H$ and one copy of $F$ and we obtain the thesis. 
3. The results follows from the previous considerations and the last part of Lemma 3.2.7.

Remark 3.6. Hence we have proved not only that a $p$-group $E$ is extraspecial if and only if it is a central product of extraspecial $p$-groups of order $p^{3}$ with amalgamated centers, but also that, if $p$ is odd, the structure of $E$ is uniquely determined by the order of $E$ and $\exp (E)$.

Moreover, in view of Corollary 3.2.4, we notice that, if $p$ is an odd prime and $\left\{G_{m}\right\}_{m}$ is the family of extraspecial groups of order $p^{2 m+1}$ and exponent $p$, then $G_{m+1} \simeq G_{m} \curlyvee H$.

\subsection{Some irreducible modules of an extraspecial group}

In this section we describe certain irreducible modules of an extraspecial $p$-group over a finite field.

Irreducible modules of extraspecial groups play an important role in the theory of representation of soluble groups. However we do not want to make the whole theory for these modules, but only to construct some of them in a special case in order to provide the basis for the examples in the next chapter. For a complete overview on this topic we refer to [3], Ch. B, $\S 9$.

If $p$ is an odd prime and $K$ is any field, then Thm. 9.16 of Ch. B, $\S 9$ of [3] provides a classification of all simple modules of an extraspecial $p$-group $E$ over a field $K$. This classification depends only on the order of $E$ and on the degree of the minimal extension of $K$ containing a primitive $p$-th root of unity. In particular this result provides the existence of $p-1$ absolutely irreducible and faithful modules when $K$ contains a primitive $p$-th root of unity.

We shall now give an explicit construction of one of these modules. We fix two odd primes $p$ and $q$, with $p$ dividing $q-1$, such that the finite field $K=\mathbb{F}_{q}$ contains a primitive $p$-th root of unity $\zeta$.

We denote by $E$ the extraspecial group of order $p^{3}$, exponent $p$ and generators $x$ and $y$ and we set $W=\left(\mathbb{F}_{q}\right)^{p}$.

Then $W$ has the structure of $E$-module via the following action: if $\left\{e_{1}, \ldots, e_{p}\right\}$ is a basis for $W$, then $x$ permutes the $e_{i}$ 's as a cycle of length $p$ and $y \cdot e_{i}=\zeta^{i} e_{i}$.

Now for every positive integer $m$ we denote by $E_{m}$ the extraspecial group of order $p^{2 m+1}$ and exponent $p$ and by $W_{m}$ the tensor product of $m$ copies of $W$. By Theorem 3.2.8, $E_{m}$ is isomorphic to a quotient of the direct product of $m$ copies of $E$, thus it acts on $W_{m}$, which can be regarded as an $E_{m}$-module of dimension $p^{m}$ over $\mathbb{F}_{q}$.

We have the following proposition.

Proposition 3.3.1. $W_{m}$ is a faithful and absolutely irreducible $E_{m}$-module. 
Proof. It is easy to see that the module $W_{m}$ is faithful for $E_{m}$.

Now, since the tensor product of absolutely irreducible modules is absolutely irreducible, we need to prove that $W$ is an absolutely irreducible $E$-module. This is equivalent to show that $\operatorname{End}_{E}(W) \simeq \mathbb{F}_{q}$. Now a matrix $C$ belongs to $\operatorname{End}_{E}(W)$ if and only if $C$ commutes with the generators of $E$. As before, we have that $E$ is generated by two elements $x$ and $y$ and the matrices associated to these generators are the $p \times p$ matrices $X$ and $Y$, where:

1. $X$ represents the cyclic permutation $(1, \ldots, p)$;

2. $Y$ is a diagonal matrix whose entries are the $p$ distinct $p$-th roots of unity.

We notice that $C \cdot Y=Y \cdot C$ if and only if $C$ is a diagonal matrix, but since we must have $C \cdot X=X \cdot C$ then $C$ must be a scalar matrix. Therefore $\operatorname{End}_{E}(W) \simeq \mathbb{F}_{q}$.

\subsection{A special property of extraspecial groups}

We end this chapter with some properties of an extraspecial group. These results seem to be ad hoc as they will be used only at the end of the next chapter, when their importance shall become evident.

The first result is about the cardinality of subgroups of extraspecial groups with a bounded number of generators. This will be used to provide an explicit bound for the local degrees of a certain infinite algebraic extension of the rationals which we shall construct in the next chapter.

Proposition 3.4.1. Let $G$ be an extraspecial group of order $p^{2 m+1}$ and let $H$ be a subgroup of $G$ with at most $n$ generators. Then $|H| \leq p^{n+1}$.

Proof. We suppose first that $H$ contains the center $Z(G)$. Then we have

$$
H / Z(G) \leq G / Z(G) \simeq(\mathbb{Z} / p \mathbb{Z})^{2 m}
$$

and $H / Z(G)$ has at most $n$ generators as a subspace of $G / Z(G)$. Hence $|H / Z(G)| \leq$ $p^{n}$, since it is elementary abelian as a group, and $|H| \leq p^{n+1}$.

Now we suppose that $Z(G) \nsubseteq H$ and we consider $K=H Z(G)$ which is a subgroup of $G$ (since $Z(G)$ is normal in $G$ ) that contains the center and has at most $n+1$ generators ( $n$ generators from $H$ and one generator from the center which is cyclic by hypothesis). With the previous argument we obtain that $|K / Z(G)| \leq p^{n+1}$ and $|K| \leq p^{n+2}$. Finally we get $|H| \leq p^{n+1}$.

The second property is about the intersection of all subgroups of an extraspecial group of bounded index. It will be used to provide subfields of $\mathbb{Q}^{\left(p^{3}\right)}$ which cannot be generated by elements of bounded degree. Before we state this result we need the following lemma, which is about maximal abelian subgroups of an extraspecial $p$-group.

Lemma 3.4.2. We let $p$ be an odd prime and $P$ be an extraspecial p-group of order $p^{2 m+1}$. Then an abelian subgroup of $P$ has order at most $p^{m+1}$. 
Proof. Let $M$ be an abelian subgroup of $P$. We can suppose $M$ to be maximal. Then $Z(P) \subseteq M$, otherwise $M^{\prime}=M Z(P)$ would be abelian and containing $M$.

We consider $W=M / Z(P)$ : it is a subspace of $V=P / Z(P)$, which is a symplectic space endowed with the form defined in Lemma 3.1.4 and, since $M$ is abelian, we have that $W \subseteq W^{\perp}$. Hence

$$
2 \operatorname{dim}(W) \leq \operatorname{dim}(W)+\operatorname{dim}\left(W^{\perp}\right)=\operatorname{dim}(V)=2 m,
$$

thus $|M|=p^{\operatorname{dim}(W)+1} \leq p^{m+1}$.

We can now state our result.

Proposition 3.4.3. Let $p$ be an odd prime and $G$ be an extraspecial p-group of order $p^{2 m+1}$. Then the intersection of all subgroups of $G$ of index at most $p^{m}$ is nontrivial.

Proof. Let $H$ be a subgroup of $G$ such that $[G: H] \leq p^{m}$, we have that $|H| \geq$ $p^{m+1}$. Either $H$ contains the center $Z(G)$ or $|H Z(G)|>p^{m+1}$ and from Lemma 3.4.2 $\mathrm{HZ}(G)$ is not abelian; therefore $H$ is also not abelian and it contains two elements which do not commute. This implies that $G^{\prime}=Z(G) \subseteq H$, since $G^{\prime}$ is cyclic of order $p$. 


\section{Chapter 4}

\section{The main theorem}

In Chapter 2 we saw that a for an infinite abelian extension of $\mathbb{Q}$ there is a list of properties which are equivalent to the property of having uniformly bounded local degrees.

The purpose of this chapter is to prove that this is not true in general. The non equivalence is entailed by the existence of fields with special properties constructed using extraspecial groups and their modules.

\subsection{Statement of the theorem}

We now state the main result which will be proved throughout this chapter.

Theorem 2. Let $K$ be an infinite algebraic Galois extension of $\mathbb{Q}$. Consider the following properties for $K$ :

(a) K has uniformly bounded local degrees;

(b) $K$ is contained in $\mathbb{Q}^{(d)}$ for any positive integer $d$;

(c) every finite subextension of $K$ can be generated by elements of bounded degree over $\mathbb{Q}$;

(d) there exists a positive integer $b$ such that, whenever $K$ can be written as a compositum of a family $\left\{K_{m}\right\}_{m}$ of finite Galois extensions, then for every $m$ the Galois group of $K_{m}$ has only minimal normal subgroups of order at most $b$.

Then:

(1) $(c) \Rightarrow(b) \Rightarrow(a)$;

(2) $(b) \Rightarrow(d)$;

and no other implication holds, that is: 
(i) $(d) \nRightarrow(b)$;

(ii) $(b) \nRightarrow(c)$;

(iii) $(a) \nRightarrow(b)$.

Remark 4.1. It is immediate to see that if $K$ satisfies (c) then it also satisfies (b) and, from Theorem 2.1.2, it follows easily that $(c) \Rightarrow(b) \Rightarrow(a)$.

In order to prove that (b) implies property (d), and that all the converse arrows are not true we need preliminary considerations. As the proof of these results is quite long, we divide it into four different parts.

\subsection{Proof of (2)}

We consider an infinite algebraic Galois extension $K$ of $\mathbb{Q}$ for which property (b) holds, that is $K$ is contained in $\mathbb{Q}^{(d)}$ for some positive integer $d$.

We can write $K$ as the compositum of all its finite Galois subextensions $\left\{K_{m}\right\}_{m}$ and we denote by $G_{m}=\operatorname{Gal}\left(K_{m} / \mathbb{Q}\right)$ its Galois group.

We want to prove that for the field $K$ property (d) holds, that is there exists a positive integer $b$ such that, for every $m$, every minimal normal subgroup of $G_{m}$ has order at most $b$. In order to do this we need the following general lemma.

Lemma 4.2.1. Let $G$ be a finite group with a minimal normal subgroup $W$ such that $|W|=m$. Suppose that $G$ is a quotient of a group $H$ where $H$ is a subgroup of a direct product $H_{1} \times \ldots \times H_{s}$. Then $\left|H_{i}\right| \geq m$ for some index $i \in\{1, \ldots, s\}$

Proof. We suppose that $G=H / N$ with $H \leq H_{1} \times \ldots \times H_{s}$ and that $\left|H_{i}\right|<m$ for every $i$. Let us denote by $\pi: H \rightarrow H / N$ the projection map. We set $\overline{H_{i}}:=H \cap 1 \times \ldots \times 1 \times H_{i} \times \ldots \times H_{r}$ and we denote by $G_{i}=\pi\left(\overline{H_{i}}\right)$ the image of $\overline{H_{i}}$ in $G$. We notice that the $G_{i}$ 's are all normal subgroups of $G$.

We want to show by induction that $W \subseteq G_{i}$ for every $i$.

For $i=1$ this holds by assumption, since $G_{1}=G$.

Suppose it is true for $G_{i-1}$. Now $W \subseteq G_{i-1}$ and $\left(G_{i-1}: G_{i}\right)<m$, thus $U:=G_{i} \cap W$ is normal and non trivial and so it contains $W$. Therefore $W \subseteq G_{i}$. In particular $W \subseteq G_{s}$, which is a contradiction, since $\left|G_{s}\right| \leq\left|H_{s}\right|<m$.

Now we are able to prove our result. In fact, suppose that $K \subseteq \mathbb{Q}^{(d)}$ for some integer $d$ and it does not satisfy property (d).

This means that there exists an increasing sequence of positive integer $\left\{c_{m}\right\}_{m}$ such that $G_{m}$ has a minimal normal subgroup of order $c_{m}$.

Thus, in view of Lemma 4.2.1, whenever $G_{m}$ is isomorphic to a quotient $H / N$ and $H$ is a subgroup of a direct product $H_{1} \times \ldots \times H_{s}$, then $\left|H_{i}\right| \geq c_{m}$ for at least one index $i$.

We fix $m$ such that $d !<c_{m}$. We notice that, since $K$ is contained in $\mathbb{Q}^{(d)}$, so is $K_{m}$, then there exist number fields $L_{1}, \ldots, L_{s}$ such that $K_{m} \subseteq L_{1} \ldots L_{s}$ and $\left[L_{i}: \mathbb{Q}\right] \leq d$. 
We denote by $L_{i}^{\text {Gal }}$ the Galois closure of $L_{i}$ in $\mathbb{Q}^{\text {alg }}$ and by $H_{i}=\operatorname{Gal}\left(L_{i}^{\text {Gal }} / \mathbb{Q}\right)$ its Galois group. We notice that $\left|H_{i}\right| \leq d$ ! $<c_{m}$ by hypothesis.

We have $K_{m} \subseteq L_{1}^{\mathrm{Gal}} \ldots L_{s}^{\mathrm{Gal}}$ and $\operatorname{Gal}\left(L_{1}^{\mathrm{Gal}} \ldots L_{s}^{\mathrm{Gal}}\right)$ is isomorphic to a subgroup of $H_{1} \times \ldots \times H_{s}$ via the restriction map.

Since $K_{m}$ is a Galois extension, then

$$
G_{m}=\operatorname{Gal}\left(K_{m} / \mathbb{Q}\right) \simeq \operatorname{Gal}\left(L_{1}^{\mathrm{Gal}} \ldots L_{s}^{\mathrm{Gal}}\right) / N
$$

for some normal subgroup $N$ of $\operatorname{Gal}\left(L_{1}^{\mathrm{Gal}} \ldots L_{s}^{\mathrm{Gal}}\right)$.

Now $\operatorname{Gal}\left(L_{1}^{\mathrm{Gal}} \ldots L_{s}^{\mathrm{Gal}}\right)$ is a subgroup of $H_{1} \times \ldots \times H_{s}$ with $\left|H_{i}\right|<c_{m}$ for every $i$, which is a contradiction. Therefore $K$ satisfies (d).

\subsection{Proof of (i)}

We now want to prove that condition (d) does not imply condition (b).

In order to do this we fix a prime number $p$ and we consider the family of finite groups $\left\{G_{m}\right\}_{m}$ where $G_{m}=\mathbb{Z} / p^{m} \mathbb{Z}$ is the cyclic group of order $p^{m}$. We choose a Galois extension $K_{m}$ of $\mathbb{Q}$ with Galois group $G_{m}$ and we denote by $K$ the compositum of all the $K_{m}$ 's.

We notice that $\exp (\operatorname{Gal}(K / \mathbb{Q}))$ is not finite, since the group $\operatorname{Gal}(K / \mathbb{Q})$ contains elements of order $p^{m}$ for every $m$. In view of Theorem $2.2 .2, K$ cannot have uniformly bounded local degrees and thus it is not contained in $\mathbb{Q}^{(d)}$.

However, for every $m$, every minimal normal subgroup of $G_{m}$ has order $p$. In fact suppose that $G_{m}$ has a minimal normal subgroup $H$ of order at least $p^{2}$. Then $H$ contains a subgroup of order $p$, which is normal in $G_{m}$; this contradicts the assumption on the minimality of $H$. Therefore $K$ satisfies property (d).

\subsection{Proof of (ii)}

We want to show that condition (b) does not imply condition (c).

We fix an odd prime number $p$ and we denote by $E$ the extraspecial group of order $p^{3}$.

We now consider an infinite family $\left\{F_{i}\right\}_{i}$ of linearly disjoint finite Galois extensions of $\mathbb{Q}$, where $\operatorname{Gal}\left(F_{i} / \mathbb{Q}\right)=E$ for every $i$ and $F_{i} \cap F_{j}=1$ for $i \neq j$.

We know that such a family exists: in fact a famous theorem of Shafarevich (see [14]) asserts that every solvable group of odd order can be realized over $\mathbb{Q}$. Now, for every integer $n$, the group $E^{n}=E \times \ldots \times E$, being a $p$-group, is solvable and hence realizable. We denote by $F$ its realization, that is $F$ is a finite Galois extension of the rationals with Galois group $\operatorname{Gal}(F / \mathbb{Q})=E^{n}$. We notice that $E^{n}$ has $n$ normal subgroups $H_{1}, \ldots, H_{n}$ isomorphic to $E^{n-1}$ whose intersection is trivial and $E^{n} / H_{i}=E$ for all $i$ 's. Thus $F^{H_{1}}, \ldots, F^{H_{n}}$ are $n$ linearly disjoint realization of $E$ over $\mathbb{Q}$ and we can make this construction for every integer $n$.

Remark 4.2. If one wants to avoid the use of Shafarevich's result, in section 5.1 .1 of Chapter 5, we will give a realization of such a family. 
Now we consider the family of finite Galois extensions $\left\{K_{m}\right\}_{m}$ constructed in the following way: for every $m$, we denote by $L_{m}$ the compositum of $m$ linearly disjoint extraspecial extensions of order $p^{3} F_{1}, \ldots, F_{m}$. By construction, we have $\operatorname{Gal}\left(L_{m} / \mathbb{Q}\right)=E \times \ldots \times E=E^{m}$. Now we consider the normal subgroup $N_{m}$ of $E^{m}$ described in Proposition 3.2.1 and we denote by $K_{m}:=L_{m}^{N_{m}}$ the subfield of $L_{m}$ fixed by this subgroup.

We have that $K_{m} / \mathbb{Q}$ is a finite Galois extension with Galois group $G_{m}=$ $E^{m} / N_{m}$ which is an extraspecial group of order $p^{2 m+1}$.

We denote by $K$ the compositum of the family $\left\{K_{m}\right\}_{m}$. By construction every field $K_{m}$ is contained in $\mathbb{Q}^{\left(p^{3}\right)}$, so $K \subseteq \mathbb{Q}^{\left(p^{3}\right)}$ and (b) holds for $K$.

However, suppose that there exists a positive integer $d$ such that, for every $m, K_{m}$ can be generated by elements of degree smaller than $d$, that is $K_{m}$ can be written as the compositum of all its subextensions of degree at most $d$ over $\mathbb{Q}$. We now fix $m$ such that $d<p^{m}$ and we denote by $\left\{H_{m, i}\right\}_{i}$ the family of all subgroups of $G_{m}$ of index at most $p^{m}$ in $G_{m}$. By the Galois correspondence, $\left\{K_{m}^{H_{m, i}}\right\}_{i}$ is the family of all subextensions of $K_{m}$ of degree at most $p^{m}$ over $\mathbb{Q}$ and we denote the compositum of this family by $F_{m}$.

We have the following situation

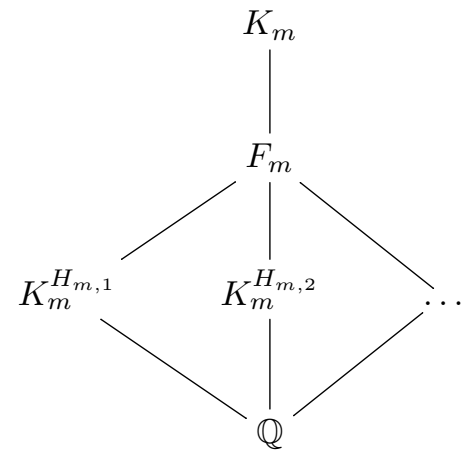

We notice that $F_{m}$ is strictly contained in $K_{m}$ since $\operatorname{Gal}\left(K_{m} / F_{m}\right)=\bigcap_{i} H_{m, i}$ and, from Proposition 3.4.3, we have

$$
Z\left(G_{m}\right) \subseteq \bigcap_{i} H_{m, i}=\operatorname{Gal}\left(K_{m} / F_{m}\right) \neq 1
$$

Therefore $K_{m}$ strictly contains the compositum of its subextensions of degree at most $d$ and $K$ does not satisfies property (c).

\subsection{Proof of (iii)}

We now want to prove that (a) does not imply (b). To do this, it suffices to show the existence of an extension $K$ of $\mathbb{Q}$ with uniformly bounded local degrees which is not contained in $\mathbb{Q}^{(d)}$ for any integer $d$.

In view of Theorem 2.2.2 and property (2), in order to construct such a field, we have to find a family of finite Galois extensions $\left\{K_{m}\right\}_{m}$ such that, 
setting $G_{m}:=\operatorname{Gal}\left(K_{m} / \mathbb{Q}\right)$, the family of groups $\left\{G_{m}\right\}_{m}$ satisfies the following conditions:

1. $\exp \left(G_{m}\right)$ is bounded by a constant which does not depend on $m$;

2. there exists a strictly increasing sequence of positive integer $\left\{c_{m}\right\}_{m}$ such that $G_{m}$ has a minimal normal subgroup of order $c_{m}$.

Then the compositum $K$ of the family of fields $\left\{K_{m}\right\}_{m}$ has the desired property.

In fact condition $(1)$ implies that $\exp (\operatorname{Gal}(K / \mathbb{Q}))$ is finite, which is equivalent to the fact that $K$ has uniformly bounded local degrees. As for condition (2), it implies that (b) does not hold for $K$.

\subsubsection{Construction of the field $K$}

We take $p$ and $q$ two odd primes such that $p$ divides $q-1$.

We denote by $E_{m}$ the extraspecial group of order $p^{2 m+1}$ and exponent $p$ and by $\mathbb{F}_{q}\left[E_{m}\right]$ its regular representation.

We recall that

$$
\mathbb{F}_{q}\left[E_{m}\right]=\left\{\sum_{e \in E_{m}} a_{e} e \mid a_{e} \in \mathbb{F}_{q}\right\}
$$

and that $E_{m}$ acts on $\mathbb{F}_{q}\left[E_{m}\right]$ by translation.

Now we consider the family of finite groups $\left\{G_{m}\right\}_{m \geq 1}$ where

$$
G_{m}=\mathbb{F}_{q}\left[E_{m}\right] \rtimes E_{m}
$$

the semidirect product being taken via the natural action of $E_{m}$ on $\mathbb{F}_{q}\left[E_{m}\right]$.

We can now prove the following lemma.

Lemma 4.5.1. The family $\left\{G_{m}\right\}_{m \geq 1}$ satisfies condition (1) with bound $p q$ and condition (2) with $c_{m}=q^{p^{m}}$.

Proof. We have that $\exp \left(G_{m}\right)=p q$ for every $m$, thus condition (1) holds.

As for condition (2), we notice that, since $q$ and $p$ are different primes, then by Maschke's theorem (see [3], Ch.A, $\S 11$, Thm. 11.5) the group algebra $\mathbb{F}_{q}\left[E_{m}\right]$ is a semisimple algebra. In particular every irreducible $E_{m}$-module over $\mathbb{F}_{q}$ appears as a direct summand of $\mathbb{F}_{q}\left[E_{m}\right]$.

We now take the absolutely irreducible $E_{m}$-module $W_{m}$ constructed in Section 3.3. By abuse of notation, we denote by $W_{m}$ a subgroup of $\mathbb{F}_{q}\left[E_{m}\right]$ isomorphic as a submodule to $W_{m}$. Finally, we denote by $H_{m}$ the subgroup of $G_{m}$ given by $H_{m}:=W_{m} \rtimes 1$.

We have that $H_{m}$ is a normal subgroup of $G_{m}$ and it is also minimal since it is isomorphic to $W_{m}$ which is irreducible as a module. Moreover it has order $\left|H_{m}\right|=q^{p^{m}}$. Thus condition (2) is true with $c_{m}=\left|H_{m}\right|=q^{p^{m}}$.

We have now constructed a family of groups which satisfies the desired properties and we want to find a family of number fields realizing these groups. Such 
a family exists: in fact the groups $G_{m}$ 's are $p q$-groups and, by Burnside's Theorem (see [3], Ch. I, §2, p.210), they are solvable. Again by Shafarevich's Theorem (see [14]) they can be realized over $\mathbb{Q}$ (recall that $p$ and $q$ are odd primes), that is there exists a family of number fields $\left\{K_{m}\right\}_{m \geq 1}$ such that, for every $m, \operatorname{Gal}\left(K_{m} / \mathbb{Q}\right)=G_{m}$ and we denote by $K$ the compositum of this family. Therefore the field $K$ satisfies (a), but not (b) of Thereom 2 .

Remark 4.3. Shafarevich's result is not necessary to prove the existence of such a family, since this will be explicitly constructed in Section 5.2 of Chapter 5.

\subsubsection{An explicit bound for the local degrees of $K$}

In view of Theorem 2.2.2, condition (1) is sufficient to prove that the constructed field $K$ has uniformly bounded local degrees; however one may be interested in knowing an effective bound for the local degrees of $K$.

We fix a prime number $\ell$ and we consider the completion of every $K_{m}$ at a prime $v$ above $\ell$, say $K_{m, v}$. The Galois group of the tame part of the extension $K_{m, v} / \mathbb{Q}_{l}$ is metabelian of order at most $(p q)^{3}$. In view of Theorem 1.6.2, the first ramification group $G_{1}$ has the following structure:

- for $\ell=q$ it is a subgroup of $\mathbb{F}_{q}\left[E_{m}\right]$ and therefore elementary abelian of order at most $q^{(p q)^{3}+2}$;

- for $\ell=p$ it is a subgroup of $E_{m}$ with at most $(p q)^{3}+2$ generators. In view of Proposition 3.4.1, $\left|G_{1}\right| \leq p^{(p q)^{3}+3}$.

- $G_{1}$ is trivial otherwise.

Now we can apply the formula given in Theorem 2.3.1 with $n=(p q)^{3}+3$ and we obtain an explicit bound for the local degrees of $K$.

This bound is very huge and it can be significantly improved via the following considerations. We fix a prime $\ell$ of $\mathbb{Q}$ and we denote by $w$ a valuation of $K$ above $\ell$ and by $v$ the restriction of $w$ to $K_{m}$. We have to discuss three cases.

1. If $\ell \neq p, q$ then, for every $m$, the extension $K_{m, v} / \mathbb{Q}_{\ell}$ is tame and it is contained in the compositum of all metabelian extensions of $\mathbb{Q}_{\ell}$ of exponent dividing $p q$, which has degree bounded by $q^{6}$.

2. If $\ell=q$ the unramified part of the extension is contained in the compositum $L^{\text {ur }}$ of all unramified extensions of $\mathbb{Q}_{q}$ of degree dividing $p q$ and this extension has degree bounded by $p q$; the tame and totally ramified part of the extension is either trivial or cyclic of degree $p$. Therefore it is contained in the compositum $L^{\text {tame }}$ of all cyclic extensions of $L^{\mathrm{ur}}$ of degree $p$, which has degree bounded again by $p^{2}$. Finally, the wild ramified part, having Galois group which is a subgroup of $\mathbb{F}_{q}\left[E_{m}\right]$ and therefore elementary abelian, is contained in the compositum of all $q$-elementary abelian extensions of $L^{\text {tame }}$, which has degree over $L^{\text {tame }}$ at most $q^{p^{3} q+2}$. Therefore the local degree of $K$ at $q$ is at most $q^{p^{3} q+3} p^{3}<q^{q^{4}+6}$. 
3. If $\ell=p$ we denote by $H_{m}$ the Galois group of the local extension $K_{m, v} / \mathbb{Q}_{p}$. Since $\operatorname{Gal}\left(K_{m} / \mathbb{Q}\right)=\mathbb{F}_{q}\left[E_{m}\right] \rtimes E_{m}$ is a $p q$-group with a unique $q$-Sylow subgroup $\mathbb{F}_{q}\left[E_{m}\right]$, which is normal, then $H_{m}$ also possesses a unique $q$ Sylow subgroup $Q_{m}$. By Schur-Zassenhaus's theorem, since $p$ and $q$ are different primes, $H_{m}=Q_{m} \rtimes P_{m}$, where $P_{m}$ is the $p$-Sylow subgroup, $Q_{m} \leq \mathbb{F}_{q}\left[E_{m}\right], P_{m} \leq E_{m}$ and the action of $P_{m}$ on $Q_{m}$ is induced by the action of $E_{m}$ on $\mathbb{F}_{q}\left[E_{m}\right]$ by restriction. Then the extension $K_{m, v}^{Q_{m}} / \mathbb{Q}_{p}$, having metabelian Galois group $P_{m}$, is contained in the compositum $L$ of all metabelian $p$-extensions of $\mathbb{Q}_{p}$, which has degree $\left[L: \mathbb{Q}_{p}\right] \leq p^{p^{2}+4} \leq$ $q^{q^{2}+4}$. Moreover $K_{m, v} / K_{m, v}^{Q_{m}}$ is an abelian $q$-extension of a $p$-adic field with $(p, q)=1$, thus it is contained in the compositum $L^{\prime}$ of all tamely ramified extensions of $L$ of degree $q$ and we have $\left[L^{\prime}: L\right] \leq q^{2}$. Then the local degree of $K$ at $p$ is bounded by $q^{q^{2}+6}$.

Summing up these results, $K$ has local degrees bounded by $q^{q^{4}+6}$.

Remark 4.4. A further improvement of the bound can be obtained using a theorem which is due to Scholz and Reichardt (see [11]). We only state this result, which is an important theorem of inverse Galois theory and it is a special case of Shafarevich's Theorem about realization of $p$-groups over $\mathbb{Q}$. One can find a modern presentation of Reichardt's proof in Serre's course on Galois theory (see [12], 2.1).

Theorem 4.5.2 (Scholz and Reichardt). Let $p$ be an odd prime. Then every p-group can be realized as a Galois group over $\mathbb{Q}$. Moreover if $G$ is a p-group of order $p^{n}$, then one can find a number field $F$ with $G a l(F / \mathbb{Q})=G$ such that:

1. $F$ is ramified above at most $n$ primes;

2. If $\ell$ is a prime of $\mathbb{Q}$ which is ramified in $F / \mathbb{Q}$ then:

(a) $\ell \equiv 1$ modulo $p$;

(b) if $\beta$ is a prime of $F$ lying over $\ell$, then the inertia group and the decomposition group associated to $\beta$ are equal.

We notice that condition (2a) implies that the field $F$ can only have tame ramification (in particular $p$ cannot ramify in $K$ ), while condition (2b) is equivalent to the fact that the local extension $F_{\beta} / \mathbb{Q}_{\ell}$ is totally ramified.

The aim is now to realize the group $G_{m}=\mathbb{F}_{q}\left[E_{m}\right] \rtimes E_{m}$ in two steps: we first realize the $p$-group $E_{m}$ using Sholz and Reichardt's theorem and we denote by $L_{m}$ its realization; now we know that there exists a Galois extension $K_{m}$ of $L_{m}$ such that $\operatorname{Gal}\left(K_{m} / \mathbb{Q}\right)=G_{m}$ (since it is an embedding problem with abelian kernel). Then, preserving the previous notation, if $\ell=p$, by construction, the extension $K_{m, v} / \mathbb{Q}_{p}$ is unramified and its degree is bounded by $q^{2}$. If $\ell \neq p, q$ the local degree is at most $q^{6}$, as we proved before.

If $\ell=q$, in view of Scholz and Reichardt's result, the extension $L_{m, v} / \mathbb{Q}_{q}$ is either trivial or totally ramified and tame. Thus it is contained in the compositum $L^{\text {tame }}$ of all totally ramified and tame extensions of $\mathbb{Q}_{q}$ of degree $p$ and this 
field has degree over $\mathbb{Q}_{q}$ bounded by $p^{2}<q^{2}$. Now the extension $K_{m, v} / L_{m, v}$ is a $q$-extension of a $q$-adic field with $q$-elementary abelian Galois group. Therefore it is contained, for every $m$, in the compositum of all $q$-elementary abelian extensions of $L^{\text {tame }}$, which has degree at most $q^{q^{2}+2}$ over $L^{\text {tame }}$.

Therefore the local degrees of the compositum $K$ of the fields $K_{m}$ 's is bounded by $q^{q^{2}+4}$. 


\section{Chapter 5}

\section{Some explicit constructions}

In Chapter 4 we saw that among the basic ingredients of the proof of the main theorem was the existence of certain Galois extensions with special properties; this was done using Shafarevich's Theorem on the realizability of solvable groups of odd order over $\mathbb{Q}$.

This chapter provides explicit realizations for the groups described in Chapter 4 . These constructions are interesting for two reasons: firstly they permit to avoid the use of Shafarevich's result. Secondly, coming back to one of the original motivations of this work, Widmer proves in paper [16] that the Northcott property for an infinite algebraic extension $K$ of $\mathbb{Q}$ is strictly related to the behavior of the discriminants of certain finite subextensions of $K$. Therefore, knowing how to concretely construct fields described in Chapter 4 could be a step towards understanding whether these extensions might have the Northcott property or not.

The chapter has the following structure: Section 5.1 is devoted to describe the realization of extraspecial $p$-groups over a field of characteristic zero containing a primitive $p$-th root of unity. This shall entail the existence of the family of number fields used in the proof of (ii) of Theorem 2, as pointed out in Remark 4.2; in Section 5.2 we use a method described by Serre in [12] to solve embedding problems with abelian kernel. This result, together with the construction of Section 5.1, shall provide a realization of the family of Galois extensions needed to prove part (iii) of the main theorem, as announced in Remark 4.3.

\subsection{Realizing extraspecial $p$-groups over number fields}

In this section we describe a method which can be used to realize extraspecial $p$-groups over fields of characteristic zero containing a primitive $p$-th root of unity. 
We saw in Chapter 3 that an extraspecial $p$-group of order $p^{2 m+1}$ is isomorphic to a quotient of the direct product of $m$ extraspecial groups of order $p^{3}$. We want to use this special property of the structure of extraspecial groups to realize them.

This is done in two steps: first we use a method described in Michailov's paper [7] to give explicit realizations of extraspecial groups of order $p^{3}$ over some number field $k$; then we obtain a Galois extension of $k$ with Galois group the extraspecial group of order $p^{2 m+1}$ as a subfield of the compositum of $m$ linearly disjoint extraspecial extensions of order $p^{3}$.

\subsubsection{Extraspecial groups of order $p^{3}$}

We let $E$ be an extraspecial group of order $p^{3}$. We want to realize $E$ over a field $k$ of characteristic zero containing a primitive $p$-th root of unit $\zeta_{p}$. In order to do this we use the method described in [7], Thm. 3.1 and Thm. 3.2.

First of all, we take a cyclic extension $k(\sqrt[p]{a}) / k$ with $\operatorname{Gal}(k(\sqrt[p]{a}) / k)=<\sigma>$ and $\sigma(\sqrt[p]{a})=\zeta \sqrt[p]{a}$. We denote by $N_{\sigma}$ the norm function of the extension $k(\sqrt[p]{a}) / k$. Now we make slightly different constructions depending on the exponent of the extraspecial group $E$.

(a) The case $\exp (E)=p$

In this case $G$ is isomorphic to the group

$$
H=<u, v \mid u^{p}=v^{p}=1,[u, v] \in Z(H)>.
$$

We now look for an element $x \in k(\sqrt[p]{a})$ such that:

1. its norm $b=N_{\sigma}(x)=\prod_{i=1}^{p} \sigma^{i}(x)$ is not a $p$-th power in $k$, that is $b \notin k^{* p}$.

2. $k(\sqrt[p]{a}) \cap k(\sqrt[p]{b})=k$, that is $k(\sqrt[p]{a}) \neq k(\sqrt[p]{b})$ (since these extensions have the same order, which is a prime, either they are equal or they intersect in $k$ ).

We notice first of all that condition (1) implies condition (2). In fact if condition (2) does not hold, then $k(\sqrt[p]{a}) \cap k(\sqrt[p]{b})=k(\sqrt[p]{a})$. Since $k$ contains a $p$-th root of unity, by Kummer theory we have that $b=a^{i} y^{p}$ for some $y \in k$ and $(i, p)=1$. Now $a \in k(\sqrt[p]{a})^{p}$ since $a=\alpha^{p}$ where $\alpha=\sqrt[p]{a}$; therefore $b=\left(\alpha^{i} y\right)^{p}$ is a $p$-th power in $k(\sqrt[p]{a})$ and its norm belongs to $k^{* p}$ and condition (1) does not hold.

Nowwe want to find the sought-for element $x$. By Chebotarev's Theorem, there exists a prime $\mathfrak{q}$ in $k$ which splits completely in $k(\sqrt[p]{a})$. We denote by $\beta_{1}, \ldots, \beta_{p}$ the distinct primes of $k(\sqrt[p]{a})$ above $\mathfrak{q}$. By Nagata's theorem on analytic independence of valuations (see [8]) the system

$$
\left\{\begin{array}{l}
v_{\beta_{1}}(X)=1 \\
v_{\beta_{i}}(X)=0 \quad \forall i \neq 1
\end{array}\right.
$$

is solvable in $k(\sqrt[p]{a})$. We take $x \in k(\sqrt[p]{a})$ which solves the system and we claim that $x$ satisfies conditions (1) and (2). 
Condition (1) holds for $x$, since $\mathfrak{q}$ exactly divides $b=N_{\sigma}(x)$, which cannot be a $p$-th power in $k$.

Now, we set $\operatorname{Gal}(k(\sqrt[p]{b}) / k)=\left\langle\tau>\right.$, with $\tau(\sqrt[p]{b})=\zeta_{p} \sqrt[p]{b}$. We have that $L=k(\sqrt[p]{a}, \sqrt[p]{b})$ is a bicyclic extension of order $p^{2}$ of $k$ and $\operatorname{Gal}(L / k)=<\bar{\sigma}, \bar{\tau}>$ with $\left.\bar{\sigma}\right|_{k(\sqrt[p]{a})}=\sigma,\left.\bar{\tau}\right|_{k(\sqrt[p]{b})}=\tau$ and $\left.\bar{\sigma}\right|_{k(\sqrt[p]{b})}=\left.\bar{\tau}\right|_{k(\sqrt[p]{a})}=\mathrm{id}$.

We now consider the element

$$
\omega=x^{p-1} \sigma\left(x^{p-2}\right) \ldots \sigma^{p-2}(x)=\frac{b^{p-1}}{\prod_{i=1}^{p-1} \sigma^{i}\left(x^{i}\right)} .
$$

We want to prove that $M=L(\sqrt[p]{\omega}) / k$ is a Galois extension with Galois group isomorphic to $H$.

The situation is the following:

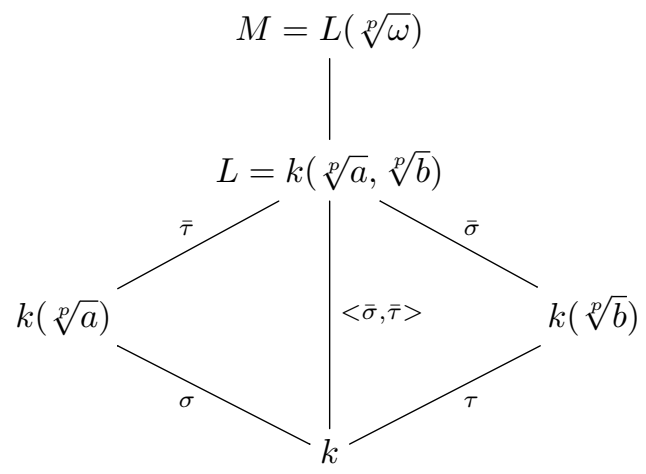

We notice that, since $\omega \in k(\sqrt[p]{a})$, then $\bar{\tau}(\omega)=\omega$. Moreover we have that

$$
\bar{\sigma}(\omega)=\sigma(\omega)=\frac{b^{p-1} \cdot b}{\prod_{i=1}^{p-1} \sigma^{i}\left(x^{i}\right) x^{p}}=\omega\left(\frac{\sqrt[p]{b}}{x}\right)^{p}
$$

hence $\bar{\sigma}(\omega) / \omega \in L^{* p}$. Thus $M / k$ is a Galois extension and we extend $\bar{\sigma}$ and $\bar{\tau}$ to $\operatorname{Gal}(M / k)$ by $\widetilde{\sigma}(\sqrt[p]{\omega})=\left(\frac{\sqrt[p]{b}}{x}\right)(\sqrt[p]{\omega})$ and $\bar{\tau}(\sqrt[p]{\omega})=\zeta_{p} \sqrt[p]{\omega}$.

Now we notice that $\omega \notin L^{* p}$. In fact, if we suppose that $\omega \in L^{* p}$, since $b \in L^{* p}$ and

$$
\omega=\frac{b^{p-1}}{\prod_{i=1}^{p-1} \sigma^{i}\left(x^{i}\right)},
$$

we get $\omega / b^{p-1}=z^{p}$ for some $z \in k(\sqrt[p]{a})^{*}$. From equality (5.2) we obtain $b=\left(\frac{\widetilde{\sigma}(z) x}{z}\right)^{p} \in k(\sqrt[p]{a})^{p}$ which is a contradiction. Therefore the extension $M / L$ is cyclic of order $p$ and $\operatorname{Gal}(M / L)=\left\langle\eta>\right.$, where $\eta(\sqrt[p]{\omega})=\zeta_{p} \sqrt[p]{\omega}$.

Now $M / k$ is a Galois extension of order $p^{3}$ and $\operatorname{Gal}(M / k)$ is generated by $\eta$, $\widetilde{\sigma}$ and $\widetilde{\tau}$. We also notice that $\eta$ commutes both with $\widetilde{\sigma}$ and $\widetilde{\tau}, \eta^{p}=\widetilde{\sigma}^{p}=\widetilde{\tau}^{p}=$ id and the relation $\widetilde{\sigma} \widetilde{\tau} \eta=\tilde{\tau} \widetilde{\sigma}$ holds. Thus $\operatorname{Gal}(M / k) \simeq H$, where the isomorphism is given by $\widetilde{\sigma} \mapsto u$ and $\widetilde{\tau} \mapsto v$. 
The case $p=2$

If $p=2$ the previous construction gives a dihedral extension of $k$.

In fact, preserving the previous notation, we have $L=k(\sqrt{a}, \sqrt{x \sigma(x)})$ with $\operatorname{Gal}(L / k)=<\bar{\sigma}, \bar{\tau}>$. Setting $M=L(\sqrt{x})$ we have $\operatorname{Gal}(M / k)=<\widetilde{\sigma}, \widetilde{\tau}>$ with $\widetilde{\sigma}(\sqrt{x})=\left(\frac{\sqrt{x \sigma(x)}}{x}\right)(\sqrt{x})$ and $\widetilde{\tau}(\sqrt{x})=-\sqrt{x}$ which satisfy the relations $\widetilde{\sigma}^{2}=\widetilde{\tau}^{2}=(\widetilde{\sigma} \widetilde{\tau})^{4}=1$

Thus $\operatorname{Gal}(M / k)$ is isomorphic to the dihedral group of order 8 .

(b) The case $\exp (E)=p^{2}$

Now $E$ is isomorphic to the group

$$
F=<u, v \mid u^{p^{2}}=v^{p}=1,[u, v]=u^{p}>.
$$

Arguing as in the previous case, we can take an element $x \in k(\sqrt[p]{a})$ such that, if $b=\zeta_{p} N_{\sigma}(x)$, the extension $k(\sqrt[p]{b})$ is cyclic and $k(\sqrt[p]{b}) \cap k(\sqrt[p]{a})=k$. We set $\operatorname{Gal}(k(\sqrt[p]{b}) / k)=<\tau>$, with $\tau(\sqrt[p]{b})=\zeta_{p} \sqrt[p]{b}$. As before, we have that $L=k(\sqrt[p]{a}, \sqrt[p]{b})$ is a bicyclic extension of order $p^{2}$ of $k$ and $\operatorname{Gal}(L / k)=<\bar{\sigma}, \bar{\tau}>$ with $\left.\bar{\sigma}\right|_{k(\sqrt[p]{a})}=\sigma,\left.\bar{\tau}\right|_{k(\sqrt[p]{b})}=\tau$ and $\left.\bar{\sigma}\right|_{k(\sqrt[p]{b})}=\left.\bar{\tau}\right|_{k(\sqrt[p]{a})}=\mathrm{id}$.

We now consider the element

$$
\omega=x^{p-1} \sigma\left(x^{p-2}\right) \ldots \sigma^{p-2}(x) \sqrt[p]{a}=\frac{b^{p-1}}{\prod_{i=1}^{p-1} \sigma^{i}\left(x^{i}\right)} \sqrt[p]{a} .
$$

and we set $M=L(\sqrt[p]{\omega})$.

As in the previous construction, $M / k$ is a Galois extension, since $\bar{\tau}(\omega)=\omega$ and $\bar{\sigma}(\omega)=\sigma(\omega)=\omega\left(\frac{p \sqrt{b}}{x}\right)^{p}$, thus we can extend $\bar{\sigma}$ and $\bar{\tau}$ to $\operatorname{Gal}(M / k)$ by $\widetilde{\sigma}(\sqrt[p]{\omega})=\left(\frac{p \sqrt[b]{b}}{x}\right)(\sqrt[p]{\omega})$ and $\bar{\tau}(\sqrt[p]{\omega})=\zeta_{p} \sqrt[p]{\omega}$. Moreover $\omega \notin L^{* p}$.

Hence we have that $M / k$ is a Galois extension of order $p^{3}$. We set $\langle\eta\rangle=$ $\operatorname{Gal}(M / L)$ with $\eta(\sqrt[p]{\omega})=\zeta_{p} \sqrt[p]{\omega}$ and we have that $\operatorname{Gal}(M / k)$ is generated by $\eta$, $\widetilde{\sigma}$ and $\widetilde{\tau}$. Since $\eta$ is a central element and the relations $\eta^{p}=\widetilde{\sigma}^{p^{2}}=\widetilde{\tau}^{p}=\mathrm{id}$, $\eta=\widetilde{\sigma}^{p}, \widetilde{\tau} \widetilde{\sigma}=\widetilde{\sigma}^{p+1} \widetilde{\tau}$ hold, then $\operatorname{Gal}(M / k) \simeq F$, where the isomorphism is given by $\widetilde{\sigma} \mapsto u$ and $\widetilde{\tau} \mapsto v$.

\section{The case $p=2$}

If $p=2$ the previous construction gives again a dihedral extension of $k$. In fact we have $L=k(\sqrt{a}, \sqrt{-x \sigma(x)})$ and $\omega=x \sqrt{a}$, thus $M=L(\sqrt{x \sqrt{a}})$. Here the extensions of $\bar{\sigma}$ and $\bar{\tau}$ to $\operatorname{Gal}(M / k)$ are given by

$$
\widetilde{\sigma}(\sqrt{x \sqrt{a}})=\left(\frac{\sqrt{-x \sigma(x)}}{x}\right)(\sqrt{x \sqrt{a}})
$$

and

$$
\widetilde{\tau}(\sqrt{x \sqrt{a}})=-\sqrt{x \sqrt{a}}
$$


We notice that $\widetilde{\sigma}^{4}=\widetilde{\tau}^{2}=(\widetilde{\sigma} \widetilde{\tau})^{2}=1$. Thus, if we set $\varepsilon=\widetilde{\sigma} \widetilde{\tau}$, we have that $\operatorname{Gal}(M / k)=<\widetilde{\sigma}, \widetilde{\tau}>=<\varepsilon, \widetilde{\tau} \mid \varepsilon^{2}=\widetilde{\tau}^{2}=(\varepsilon \widetilde{\tau})^{4}=1>$ is isomorphic to the dihedral group of order 8 .

Remark 5.1. We have seen that for $p=2$ the above constructions give a realization of the dihedral group over $k$. This comes from the fact that actually we have realized the groups

$$
(\mathbb{Z} / p \mathbb{Z})^{2} \rtimes \mathbb{Z} / p \mathbb{Z}
$$

and

$$
\left(\mathbb{Z} / p^{2} \mathbb{Z}\right) \rtimes \mathbb{Z} / p \mathbb{Z}
$$

described in Proposition 3.2.6, which are isomorphic to the extraspecial groups $H$ and $F$. However, for $p=2$, the above groups are both isomorphic to the dihedral group of order 8 .

\section{The quaternion group}

One could ask whether a similar technique can be used in order to realize the quaternion group $Q$ of order 8, but the answer is negative, since $Q$ cannot be written as a semidirect product of two groups.

Nevertheless we can use a method described by Fujisaki in [5] to give an elementary realization of the quaternion group of order 8 over any field of characteristic zero.

We let $k$ be a field of characteristic 0 and we take two elements $m, n \in k^{*} \backslash k^{* 2}$ of the form $m=p^{2}+q^{2}+r^{2}$ and $n=p^{2}+q^{2}$, with $p, q, r \in k, p q r \neq 0$ and $m n \notin k^{* 2}$, such that the extension $L=k(\sqrt{m}, \sqrt{n})$ is a biquadratic extension of $k$. We set

$$
H=\operatorname{Gal}(L / k)=\left\{\sigma_{1}, \sigma_{2}, \sigma_{3}, \sigma_{4}\right\} \simeq(\mathbb{Z} / 2 \mathbb{Z})^{2}
$$

where

$$
\begin{array}{lll}
\sigma_{1}:(\sqrt{m}, \sqrt{n}) \mapsto(\sqrt{m}, \sqrt{n}), & \sigma_{2}:(\sqrt{m}, \sqrt{n}) \mapsto(-\sqrt{m}, \sqrt{n}), \\
\sigma_{3}:(\sqrt{m}, \sqrt{n}) \mapsto(\sqrt{m},-\sqrt{n}), & \sigma_{4}:(\sqrt{m}, \sqrt{n}) \mapsto(-\sqrt{m},-\sqrt{n}) .
\end{array}
$$

Now we consider the element

$$
\omega=\sqrt{m n}(\sqrt{m}+\sqrt{n})(\sqrt{n}+p) \in L
$$

We set $M=L(\sqrt{\omega})$ and we let $\alpha_{i}: M \rightarrow k^{\text {alg }}$ denote a fixed extension of $\sigma_{i}$ to 
$M$. We have the following situation:

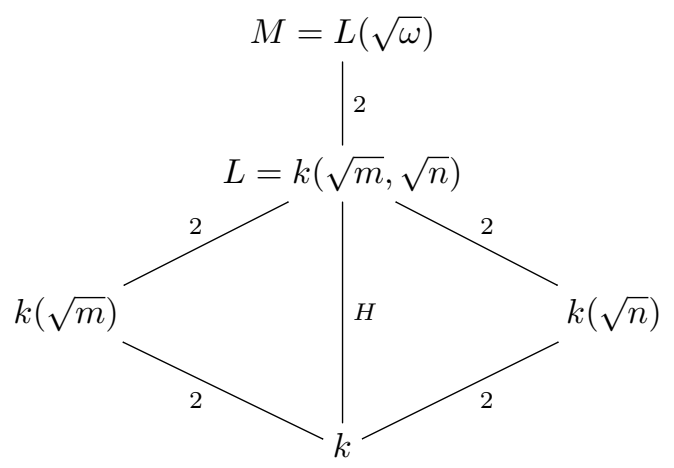

Some easy calculations show that $M / k$ is a Galois extension since $\sigma_{i}(\omega) / \omega \in$ $L^{2}$ for all $i$ 's and, in particular, we can take

$\alpha_{1}(\sqrt{\omega})=e_{1} \sqrt{\omega}$

$\alpha_{2}(\sqrt{\omega})=e_{2}\left(\frac{\sqrt{m}-\sqrt{n}}{r}\right) \sqrt{\omega}$

$\alpha_{3}(\sqrt{\omega})=e_{3}\left(\frac{\sqrt{m}-\sqrt{n}}{r}\right)\left(\frac{\sqrt{n}-p}{q}\right) \sqrt{\omega}$,

$\alpha_{4}(\sqrt{\omega})=e_{4}\left(\frac{\sqrt{n}-p}{q}\right) \sqrt{\omega}$

where $e_{i} \in\{ \pm 1\}$ are signs depending on $\alpha_{i}$.

Moreover we have that $\left.\alpha_{i}^{2}\right|_{L}=\sigma_{i}^{2}=\mathrm{id}$ and $\alpha_{i}^{2}(\sqrt{\omega})=-\sqrt{\omega}$ for all $i$ 's. Thus $\sqrt{\omega} \notin L$, otherwise it would be fixed by $\alpha_{i}^{2}$.

So we proved that the extension $M / k$ is a Galois extension of degree 8. Let us set $G=\operatorname{Gal}(M / k)$. It is easy to see, choosing $e_{1}=-1$, that

$$
G=\left\{i d, \alpha_{1}, \alpha_{2}, \alpha_{2}^{3}, \alpha_{3}, \alpha_{3}^{3}, \alpha_{4}, \alpha_{4}^{3}\right\}
$$

since they are all distinct elements.

Replacing $\alpha_{i}$ by $\alpha_{i}^{3}$, we can suppose that $e_{i}=1$ for $i \in\{2,3,4\}$. Then we have the following relations: $\alpha_{i}^{4}=1$ for all $i$ 's, $\alpha_{i}^{2}=\alpha_{1}$ for $i \in\{2,3,4\}$, $\alpha_{3} \alpha_{2}=\alpha_{4}, \alpha_{4} \alpha_{3}=\alpha_{2}, \alpha_{2} \alpha_{4}=\alpha_{3}$ e $\alpha_{3} \alpha_{2} \alpha_{3}^{-1}=\alpha_{2}^{3}=\alpha_{2}^{-1}$.

These relations shows that

$$
G=\operatorname{Gal}(M / k)=<\alpha_{2}, \alpha_{3} \mid \alpha_{2}^{2}=\alpha_{3}^{2}, \alpha_{2}^{4}=\mathrm{id}, \alpha_{3} \alpha_{2} \alpha_{3}^{-1}=\alpha_{2}^{-1}>
$$

is isomorphic to the quaternion group $Q$.

\subsubsection{Extraspecial groups of any order and exponent $p$}

After a brief overview on the realization of extraspecial groups of order $p^{3}$, we focus on extraspecial groups of order $p^{2 m+1}$ and exponent $p$. We want to prove the following theorem.

Theorem 5.1.1. Let $k$ be a field of characteristic zero containing a primitive $p$ th root of unity $\zeta_{p}$. Let $a_{1}, b_{1}, \ldots, a_{m}, b_{m} \in k$ be elements defined in the following way. 
(i) For every $i$ the element $a_{i}$ is not a $p$-th power in $k$ and the ideal $\left(a_{i}\right)$ is prime to the ideals $\left(a_{j}\right)$ and $\left(b_{j}\right)$ for every $j<i$.

(ii) Denote by $\sigma_{i}$ the generator of $\operatorname{Gal}\left(k\left(\sqrt[p]{a_{i}}\right) / k\right)$ such that $\sigma_{i}\left(\sqrt[p]{a_{i}}\right)=\zeta_{p} \sqrt[p]{a_{i}}$. Let $\mathfrak{q}_{i}$ be a prime of $k$ which splits completely in the extension $k\left(\sqrt[p]{a_{i}}\right) / k$ and such that $\mathfrak{q}_{i} \neq \mathfrak{q}_{j}$ for every $j<i$. Denote by $\beta_{1}^{i}, \ldots, \beta_{p}^{i}$ the primes of $k\left(\sqrt[p]{a_{i}}\right)$ above $\mathfrak{q}_{i}$ and by $S_{i}$ the set of primes of $k\left(\sqrt[p]{a_{i}}\right)$ lying above $p$ and above the primes of $k$ dividing $\left(a_{j}\right)$ or $\left(b_{j}\right)$ for $j<i$. Let $x_{i} \in k\left(\sqrt[p]{a_{i}}\right)$ be a solution of the system

$$
\left\{\begin{array}{c}
v_{\beta_{1}^{i}}(X)=1 \\
v_{\beta_{j}^{i}}(X)=0 \quad \forall j \neq 1 \\
v_{\beta}(X)=0 \quad \forall \beta \in S_{i} .
\end{array}\right.
$$

and set $b_{i}:=N_{\sigma_{i}}\left(x_{i}\right)$.

Finally consider the elements $\omega_{1}, \ldots, \omega_{m}$ defined as

$$
\omega_{i}:=\frac{b_{i}^{p-1}}{\prod_{j=i}^{p-1} \sigma_{i}^{j}\left(x_{i}^{j}\right)} .
$$

Then the extension $k\left(\sqrt[p]{a_{1}}, \sqrt[p]{b_{1}}, \ldots, \sqrt[p]{a_{m}}, \sqrt[p]{b_{m}}, \sqrt[p]{\omega_{1} \cdots \omega_{m}}\right) / k$ is a Galois extension with Galois group the extraspecial group of order $p^{2 m+1}$ and exponent $p$.

Proof. The first part of property (1) follows from the construction made in Section 5.1.1. In fact, setting

$$
M_{i}:=k\left(\sqrt[p]{a_{i}}, \sqrt[p]{b_{i}}, \sqrt[p]{\omega_{i}}\right)
$$

the extensions $M_{i} / k$ are Galois extensions with Galois group $H_{i}$ which is an extraspecial group of order $p^{3}$ and exponent $p$.

We want to prove that the extensions $M_{i}$ 's are linearly disjoint and that property (2) holds. We prove the result by induction on $m$. The theorem is trivially true for $i=1$.

We suppose the theorem to be true for $i<m$ and we want to prove that the extension $M_{m}$ is linearly disjoint from $M_{i}$ for every $i<m$. Since the only primes of $k$ which ramify in the extensions $k\left(\sqrt[p]{a_{i}}\right)$ and $k\left(\sqrt[p]{b_{i}}\right)$ are those above $p$ and those diving the ideals $\left(a_{i}\right)$ and $\left(b_{i}\right)$ respectively, condition $(i)$ ensures that

$$
k\left(\sqrt[p]{a_{m}}\right) \cap k\left(\sqrt[p]{a_{i}}\right)=k\left(\sqrt[p]{a_{m}}\right) \cap k\left(\sqrt[p]{b_{i}}\right)=k
$$

for every $i<m$. Since $x_{m}$ satisfies the system (5.3), we also have

$$
k\left(\sqrt[p]{a_{m}}\right) \cap k\left(\sqrt[p]{b_{m}}\right)=k .
$$

Moreover we notice that if $\beta$ is a prime of $k$ above $p$ or diving $\left(a_{i}\right)$ or $\left(b_{i}\right)$ for $i<m$, then $\beta$ does not divide $\left(b_{m}\right)$, otherwise $\left(x_{m}\right)$ would be divisible by some 
elements of $S_{m}$. Using the previous arguments we get that $k\left(\sqrt[p]{a_{m}}, \sqrt[p]{b_{m}}\right) \cap$ $k\left(\sqrt[p]{a_{i}}, \sqrt[p]{b_{i}}\right)=k$ for every $i<m$.

We notice that an extraspecial group of order $p^{3}$ has a unique normal subgroup of order $p$, which is given by the center; then the extension $k\left(\sqrt[p]{a_{m}}, \sqrt[p]{b_{m}}\right)$ is the unique $p$-elementary subextension of $M_{m}$ of order $p^{2}$. Therefore the extensions $M_{1}, \ldots, M_{m}$ are linearly disjoint.

We now want to prove condition (2). We denote by $k_{m}$ the compositum of the fields $M_{1}, \ldots, M_{m}$. Then $\operatorname{Gal}\left(k_{m} / k\right)=H_{1} \times \ldots \times H_{m}$ and the field

$$
M=k\left(\sqrt[p]{a_{1}}, \sqrt[p]{b_{1}}, \ldots, \sqrt[p]{a_{m}}, \sqrt[p]{b_{m}}, \sqrt[p]{\omega_{1} \ldots \omega_{m}}\right)
$$

is a subfield of $k_{m}$.

We consider the subgroup $N_{m}$ of $H_{1} \times \ldots \times H_{m}$ defined by

$$
N_{m}=\left\{\left(z_{1}^{a_{1}}, \ldots, z_{m}^{a_{m}}\right) \mid a_{1}+\ldots+a_{m} \equiv 0 \quad \bmod p\right\}
$$

where $Z\left(H_{i}\right)=<z_{i}>$. From previous considerations, the element $z_{i}$ acts in the following way:

- $z_{i}\left(\sqrt[p]{\omega_{i}}\right)=\zeta_{p} \sqrt[p]{\omega_{i}}$

- $z_{i}\left(\sqrt[p]{a_{i}}\right)=\sqrt[p]{a_{i}}$

- $z_{i}\left(\sqrt[p]{b_{i}}\right)=\sqrt[p]{b_{i}}$.

In view of the results of Chapter 3 , the quotient $\left(H_{1} \times \ldots \times H_{m}\right) / N_{m}$ is an extraspecial group of order $p^{2 m+1}$ and exponent $p$; therefore, by the Galois correspondence, the extension $k_{m}^{N_{m}} / k$ is an extraspecial extension of order $p^{2 m+1}$ and exponent $p$. We claim that $M=k_{m}^{N_{m}}$.

The field

$$
k\left(\sqrt[p]{a_{1}}, \sqrt[p]{b_{1}}, \ldots, \sqrt[p]{a_{m}}, \sqrt[p]{b_{m}}\right)
$$

has degree $p^{2 m}$ over $k$ and is contained in $k_{m}^{N_{m}}$, since it is fixed by every element of $N_{m}$. Thus $k_{m}^{N_{m}}$ must be a $p$-extension of this field.

If we take $n=\left(z_{1}^{a_{1}}, \ldots, z_{m}^{a_{m}}\right) \in N_{m}$, we have

$$
n\left(\sqrt[p]{\omega_{1} \cdot \ldots \cdot \omega_{m}}\right)=\zeta_{p}{ }^{a_{1}+\ldots+a_{m}} \sqrt[p]{\omega_{1} \cdot \ldots \cdot \omega_{m}}=\sqrt[p]{\omega_{1} \cdot \ldots \cdot \omega_{m}}
$$

since $\sum_{i=1}^{m} a_{i} \equiv 0 \bmod p$ for $n \in N_{m}$.

Therefore $M \subseteq k_{m}^{N_{m}}$ and, since they have the same degree over $k$, they are equal.

\subsection{Extension by the group algebra}

This section is meant to provide an explicit realization of fields used in the proof of part (iii) of Theorem 2, as announced in Remark 4.3. 
To do this we will resort to considerations from Section 3.3 on irreducible modules of extraspecial groups over certain finite fields, the explicit realization of extraspecial groups given in the previous section and a method by Serre.

We fix two odd different primes $p$ and $q$, with $p$ dividing $q-1$. We consider the family of groups $\left\{E_{m}\right\}_{m}$, where $E_{m}$ is the extraspecial $p$-group of order $p^{2 m+1}$ and exponent $p$ and we denote by $\mathbb{F}_{q}\left[E_{m}\right]$ the group algebra of $E_{m}$ over $\mathbb{F}_{q}$.

We shall now use the method described in Serre's book [12], p.18, to prove that if $F$ is a number field containing a $q$-th root of unity, $L_{m} / F$ is a Galois extension with Galois group $E_{m}$ and $G_{m}$ is the semidirect product $G_{m}=$ $\mathbb{F}_{q}\left[E_{m}\right] \rtimes E_{m}$, then the embedding problem for $L_{m} / F$ and for

$$
1 \rightarrow \mathbb{F}_{q}\left[E_{m}\right] \rightarrow G_{m} \rightarrow E_{m} \rightarrow 1
$$

has a solution, that is, there exists a Galois extension $K_{m}$ of $L_{m}$ such that:

1. $\operatorname{Gal}\left(K_{m} / L_{m}\right)=\mathbb{F}_{q}\left[E_{m}\right]$;

2. $K_{m} / F$ is Galois with Galois group isomorphic to $G_{m}$.

In order to construct $K_{m}$, we take a place $v$ of $F$ which splits completely in $L_{m}$ and we consider a place $w$ of $L_{m}$ above $v$. Now we choose an element $x \in L_{m}$ such that $x$ has $w$-adic valuation 1 , but $w_{i}$-adic valuation 0 for every $w_{i} \neq w$ above $v$.

We consider the set $\Delta:=\left\{\sqrt[q]{\sigma(x)} \mid \sigma \in \operatorname{Gal}\left(L_{m} / F\right)=E_{m}\right\}$ and we set $K_{m}=L_{m}(\Delta)$. Then the extension $K_{m} / L_{m}$ is a Galois extension with Galois group isomorphic to $\mathbb{F}_{q}\left[E_{m}\right]$. In fact we consider the map

$$
\begin{gathered}
\lambda: \mathbb{F}_{q}\left[E_{m}\right] \rightarrow \operatorname{Gal}\left(K_{m} / L_{m}\right) \\
\sum_{\sigma \in E_{m}} a_{\sigma} \sigma \longmapsto \tau
\end{gathered}
$$

where $\tau$ is defined as $\tau(\sqrt[q]{\sigma(x)})=\zeta_{q}^{a_{\sigma}} \sqrt[q]{\sigma(x)}$. Then $\lambda$ is clearly injective and surjective and it is the sought for isomorphism.

Moreover the extension $K_{m} / F$ is a Galois extension: in fact for every $\sigma \in$ $\operatorname{Gal}\left(L_{m} / F\right)=E_{m}$ and every embedding $\widetilde{\sigma}: K_{m} \rightarrow F^{\text {alg }}$ with $\left.\tilde{\sigma}\right|_{L_{m}}=\sigma$ we have that, by construction, $\widetilde{\sigma}$ is an automorphism of $K_{m}$. Finally the Galois $\operatorname{group} \operatorname{Gal}\left(K_{m} / F\right)$ is isomorphic to the semidirect product $\mathbb{F}_{q}\left[E_{m}\right] \rtimes E_{m}$, with $E_{m}$ acting on $\mathbb{F}_{q}\left[E_{m}\right]$ by translation.

Now we set $F=\mathbb{Q}\left(\zeta_{p}, \zeta_{q}\right)$ and we take $L_{m}$ to be the extension with Galois $\operatorname{group} \operatorname{Gal}\left(L_{m} / F\right)=E_{m}$ the extraspecial group of order $p^{2 m+1}$ and exponent $p$ constructed in section 5.1.2. Then we apply the above construction to find a family of number fields $\left\{K_{m}\right\}_{m}$ with Galois groups $\operatorname{Gal}\left(K_{m} / \mathbb{Q}\left(\zeta_{p}, \zeta_{q}\right)\right)=$ $\mathbb{F}_{q}\left[E_{m}\right] \rtimes E_{m}$. In view of Theorem 2, part (iii), the compositum $K$ of this family is an infinite algebraic extension of $\mathbb{Q}$ with uniformly bounded local degrees which is not contained in $\mathbb{Q}^{(d)}$ for any positive integer $d$. 


\section{Bibliography}

[1] E. Bombieri, W. Gubler, Heights in Diophantine Geometry. Cambridge Univ. Press, 2006.

[2] E. Bombieri, U. Zannier, A note on heights in certain infinite extensions of $\mathbb{Q}$. Rend. Mat. Acc. Lincei, 12, 2001, pp. 5-14.

[3] K. Doerk, T. Hawkes, Finite Solvable Groups, De Gruyter, Berlin, 1992.

[4] I. Fesenko, S. Vostokov, Local fields and their extensions, AMS, Providence, R.I., second edition, 2002.

[5] G. Fujisaki, An elementary construction of Galois quaternion extension, Proc. Japan Acad., 66, Ser.A, 1990.

[6] M. Krasner, Nombre des extensions d'un degré donné d'un corps §-adique, Les Tendances Géométriques en Algèbre et Théorie des Nombres, Ed. CNRS, Paris, 1966, pp. 143-169.

[7] I. Michailov, Four non-abelian groups of order $p^{4}$ as Galois groups, J. Algebra 307, 2007, pp. 287-299.

[8] M. Nagata, Local Rings, Interscience, New York, 1962.

[9] W. Narkiewicz, Elementary and Analytic Theory of Algebraic Numbers, Springer-Verlag, Berlin, 1990.

[10] J. Neukirch, Algebraic number theory. Springer-Verlag, Berlin/Heidelberg, 1999.

[11] H. Reichardt, Konstruktion von Zahlkörpern mit gegebener Galoisgruppe von Primzahlpotenzordnung. Journal für die reine und angewandte Mathematik, 177, 1937, pp. 1-5.

[12] J-P. Serre, Topics in Galois Theory, Research notes in Mathematics, Jones and Bartlett Publishers, 1992.

[13] I. R. Shafarevich, On p-extensions, AMS. Transl., Ser.2 4, 1956, pp. 59-72. 
[14] I. R. Shafarevich, Construction of fields of algebraic numbers with given solvable Galois group, Izv. Akad. Nauk SSSR Ser. Mat., 18:6, 1954, pp. 525578.

[15] M. R. Vaughan-Lee, The restricted Burnside problem, Second Ed., Oxford University Press, 1993.

[16] M. Widmer On certain infinite extensions of the rationals with Northcott property, Monatsh. Math., 2009. 